\title{
Dirichlet and Neumann Problems Related to Nonlinear Elliptic Systems: Solvability, Multiple Solutions, Solutions with Positive Components
}

\author{
Luisa Toscano and Speranza Toscano \\ Department of Mathematics and Applications, "R. Caccioppoli.," University of Naples "Federico II", \\ Via Claudio 21, 80125 Naples, Italy \\ Correspondence should be addressed to Luisa Toscano, luisatoscano@libero.it
}

Received 1 February 2012; Accepted 2 April 2012

Academic Editor: D. O’Regan

Copyright (C) 2012 L. Toscano and S. Toscano. This is an open access article distributed under the Creative Commons Attribution License, which permits unrestricted use, distribution, and reproduction in any medium, provided the original work is properly cited.

We study the solvability of Dirichlet and Neumann problems for different classes of nonlinear elliptic systems depending on parameters and with nonmonotone operators, using existence theorems related to a general system of variational equations in a reflexive Banach space. We also point out some regularity properties and the sign of the found solutions components. We often prove the existence of at least two different solutions with positive components.

\section{Introduction}

In this paper, we present some significant applications of the results got in [1] to Dirichlet problems (Section 2) of the type:

$$
\begin{array}{r}
-\operatorname{div}\left(A_{i}\left(x, u_{1}, \ldots, u_{n}, \nabla u_{1}, \ldots, \nabla u_{n}\right)\right) \\
=\lambda_{i} b_{i}\left|u_{i}\right|^{p-2} u_{i}+d_{i}\left(x, u_{1}, \ldots, u_{n}, \nabla u_{1}, \ldots, \nabla u_{n}\right)+f_{i} \quad \text { in } \Omega, \\
u_{i}=0 \quad \text { on } \partial \Omega \text { as } i=1, \ldots, n,
\end{array}
$$

and to Neumann problems (Section 3) of the type:

$$
\begin{aligned}
& -\operatorname{div}\left(A_{i}\left(x, u_{1}, \ldots, u_{n}, \nabla u_{1}, \ldots, \nabla u_{n}\right)\right) \\
& \quad=\lambda_{i} b_{i}\left|u_{i}\right|^{p-2} u_{i}+d_{i}\left(x, u_{1}, \ldots, u_{n}, \nabla u_{1}, \ldots, \nabla u_{n}\right)+f_{i} \quad \text { in } \Omega,
\end{aligned}
$$




$$
\begin{aligned}
& A_{i}\left(x, u_{1}, \ldots, u_{n}, \nabla u_{1}, \ldots, \nabla u_{n}\right) \cdot \mathcal{v} \\
& \quad=\mu_{i} \widehat{b}_{i}\left|u_{i}\right|^{p-2} u_{i}+\widehat{d}_{i}\left(x, u_{1}, \ldots, u_{n}, \nabla u_{1}, \ldots, \nabla u_{n}\right)+\widehat{f}_{i} \quad \text { on } \partial \Omega \quad \text { as } i=1, \ldots, n,
\end{aligned}
$$

where $n \geq 1, \lambda_{i}, \mu_{i}$ are real parameters, $\Omega$ is a bounded connected open set of $R^{N}$ with regular boundary $\partial \Omega$, and $v$ is the outward orthogonal unitary vector to $\partial \Omega$.

The study deals with the solvability of the problems, the existence of multiple solutions with all the components not identically equal to zero and, in the homogeneous case, the existence of solutions with positive components, bounded and locally Hölderian with their first derivatives. It is suitable to recall the problem studied in [1] with some notations and hypotheses.

Let $W_{1}, \ldots, W_{n}$ real reflexive Banach spaces $(n \geq 1)$. Let $W$ be the product space $X_{\ell=1}^{n} W_{\ell}$. Let $\|\cdot\|$ be the norm on $W,\|\cdot\|_{*}$ the norm on $W^{*}$ (dual space of $W$ ), and $\langle\cdot, \cdot\rangle_{\ell}($ resp. $\langle\langle\cdot, \cdot\rangle\rangle)$ the duality between $W_{\ell}^{*}$ (dual space of $\left.W_{\ell}\right)$ and $W_{\ell}\left(\right.$ resp. $W^{*}$ and $\left.W\right)$. Let us denote by " $\partial$ " Fréchet differential operator and by " $\partial_{u_{\ell}}$ " Fréchet differential operator with respect to $u_{\ell}$. Let $A \not \equiv 0$ and $D_{j} \not \equiv 0(j=1, \ldots, m ; m \geq 1)$ be real functionals defined in $W, B_{\ell}$ and $\widehat{B}_{\ell}(\ell=1, \ldots, n)$ real functionals defined in $W_{\ell}$ satisfying the conditions:

$\left(i_{11}\right) \quad A$ is lower weakly semicontinuous in $W$ and $C^{1}(W \backslash\{0\})$,

$B_{\ell}$ and $\widehat{B}_{\ell}$ are weakly continuous in $W_{\ell}$ and $C^{1}\left(W_{\ell}\right)$,

$\exists p>1: A(t v)=t^{p} A(v)$ for all $t \geq 0$ and for all $v \in W, B_{\ell}\left(t v_{\ell}\right)=t^{p} B_{\ell}\left(v_{\ell}\right)$ and $\widehat{B}_{\ell}\left(t v_{\ell}\right)=t^{p} \widehat{B}_{\ell}\left(v_{\ell}\right)$ for all $t \geq 0$ and for all $v_{\ell} \in W_{\ell}$;

$\left(i_{12}\right) \quad D_{j}$ is weakly continuous in $W$ and $C^{1}(W \backslash\{0\}), \exists q_{j}>1$ :

$$
D_{j}(t v)=t^{q_{j}} D_{j}(v) \text { for all } t \geq 0 \text { and for all } v \in W, 1<q_{1}<\cdots<q_{m} \text { if } m>1 \text {. }
$$
problem.

Let $F=\left(F_{1}, \ldots, F_{n}\right)$ with $F_{\ell} \in W_{\ell}{ }^{*}, \lambda_{\ell}$ and $\mu_{\ell} \in R$; let us consider the following

Problem $(P)$. Find $u=\left(u_{1}, \ldots, u_{n}\right) \in W \backslash\{0\}$ such that

$$
\begin{aligned}
\left\langle\partial_{u_{i}} A(u), v_{i}\right\rangle_{i}=\lambda_{i}\left\langle\partial B_{i}\left(u_{i}\right), v_{i}\right\rangle_{i}+\mu_{i}\left\langle\partial \widehat{B}_{i}\left(u_{i}\right), v_{i}\right\rangle_{i}+ & \sum_{j=1}^{m}\left\langle\partial_{u_{i}} D_{j}(u), v_{i}\right\rangle_{i}+\left\langle F_{i}, v_{i}\right\rangle_{i} \\
& \forall i \in\{1, \ldots, n\}, \quad \forall v_{i} \in W_{i}
\end{aligned}
$$

Obviously Problem (P) means to find the critical points $u \in W \backslash\{0\}$ of the Euler functional:

$$
E(v)=A(v)-\sum_{\ell=1}^{n}\left[\lambda_{\ell} B_{\ell}\left(v_{\ell}\right)+\mu_{\ell} \widehat{B}_{\ell}\left(v_{\ell}\right)\right]-\sum_{j=1}^{m} D_{j}(v)-\langle\langle F, v\rangle\rangle \quad \forall v=\left(v_{1}, \ldots, v_{n}\right) \in W
$$

where $\langle\langle F, v\rangle\rangle=\sum_{\ell=1}^{n}\left\langle F_{\ell}, v_{\ell}\right\rangle_{\ell}$. 
Let us set

$$
\begin{aligned}
& H_{\lambda \mu}(v)=A(v)-\sum_{\ell=1}^{n}\left[\lambda_{\ell} B_{\ell}\left(v_{\ell}\right)+\mu_{\ell} \widehat{B}_{\ell}\left(v_{\ell}\right)\right] \\
& \forall v=\left(v_{1}, \ldots, v_{n}\right) \in W, \quad \forall \lambda=\left(\lambda_{1}, \ldots, \lambda_{n}\right), \quad \mu=\left(\mu_{1}, \ldots, \mu_{n}\right) \in R^{n}, \\
& S_{\lambda \mu}=\left\{v \in W: H_{\lambda \mu}(v)=1\right\}, \quad V_{\lambda \mu}^{-}=\left\{v \in W: H_{\lambda \mu}(v)<0\right\}, \quad \text { as } m_{1}=1, \ldots, m \\
& V^{+}\left(D_{m_{1}}, \ldots, D_{m}\right)=\left\{v \in W: \sum_{j=m_{1}}^{m} D_{j}(v)>0\right\}, \\
& S^{+}\left(D_{1}, \ldots, D_{m}\right)=\left\{v \in W: \sum_{j=1}^{m} D_{j}(v)=1\right\}, \\
& S\left(D_{j}\right)=\left\{v \in W: D_{j}(v)=-1\right\}, \quad V^{+}(F)=\{v \in W:\langle\langle F, v\rangle\rangle>0\} .
\end{aligned}
$$

About Problem $(\mathrm{P})$, using Lagrange multipliers and the "fibering method," different existence theorems have been proved in [1]. They base on one of the following hypotheses:

$\left(i_{13}\right) \exists c(\lambda, \mu)>0:\|v\|^{p} \leq c(\lambda, \mu) H_{\lambda \mu}(v)$ for all $v \in W$;

$\left(i_{14}\right) \exists c(\lambda, \mu)>0:\|v\|^{p} \leq c(\lambda, \mu) H_{\lambda \mu}(v)$ for all $v \in V^{+}\left(D_{m}\right)$ (if $V^{+}\left(D_{m}\right) \neq \emptyset$ );

$\left(i_{15}\right) \exists m_{1} \in\{1, \ldots, m\}: V_{\lambda \mu}^{-} \cap S\left(D_{m_{1}}\right)$ is not empty and bounded in $\mathrm{W}$.

Remark 1.1. In this paper, we use some existence theorems ([1], Theorems 2.1, 2.2, 3.1, and 3.2), in which as $n>1$, in relation to a set $\mathfrak{F} \subseteq S_{\lambda \mu}$, we suppose

$\left(i_{16}^{h}\right)$ for each $v=\left(v_{1}, \ldots, v_{n}\right) \in \mathfrak{F}$ with $v_{h}=0$, there exist $\bar{v}_{h} \in W_{h} \backslash\{0\}$ and the real functions $\phi_{1}, \ldots, \phi_{n}$ such that $\phi_{h} \in C^{0}([0,1]) \cap C^{1}\left(\left[0,1[)\right.\right.$ and $\phi_{h}(1)=0$, $\phi_{\ell} \in C^{1}([0,1])$ and $\phi_{\ell}(1)=1$ as $\ell \neq h, v(s)=\left(\phi_{1}(s) v_{1}, \ldots, \phi_{h}(s) \bar{v}_{h}, \ldots, \phi_{n}(s) v_{n}\right) \in \mathfrak{F}$ for all $s \in\left[s_{0}, 1\right]\left(0 \leq s_{0}<1\right), \lim _{s \rightarrow 1^{-}}(d / d s) D_{j}(v(s))<+\infty$ for all $j \in$ $\{1, \ldots, m\}, \lim _{s \rightarrow 1^{-}}(d / d s) D_{j}(v(s))=-\infty$ for some $j \in\{1, \ldots, m\}$.

The condition $\left(i_{16}^{h}\right)$ assures that for the solutions $u=\left(u_{1}, \ldots, u_{n}\right)$ of Problem $(\mathrm{P})$, found with the method used in the recalled theorems, we have $u_{h} \neq 0$ if $F_{h} \equiv 0$.

Before showing Dirichlet problems (including the problem studied in [2] by Drábek and Pohozaev when $n=1$ and $m=1$ ) we give Propositions 2.2-2.6 which show some cases in which hypotheses $\left(i_{13}\right)-\left(i_{15}\right)$ hold. These propositions are based on the comparison between the parameters $\lambda_{i}$ with suitable eigenvalues connected to $p$-Laplacian. About Neumann problems (including the one studied in [3] by Pohozaev and Veron when $n=1$ ) the same question is solved by Propositions 3.1-3.5 in which the parameters $\lambda_{i}$ and $\mu_{i}$ have compared with zero. Finally, the results in Appendix are very useful: Propositions A.1 and A.2 in order to get condition $\left(i_{16}^{h}\right)$, Propositions A.3 and A.4 to get qualitative properties of the solutions and the positive sign of the components of the found solutions.

\section{Dirichlet Problems}

Let $\Omega \subseteq R^{N}$ be an open, bounded, connected and $C^{2, \beta}$ set with $0<\beta \leq 1$. Let $|\cdot|_{N}$ the Lebesgue measure on $R^{N}, 1<p<\infty, \tilde{p}=N p /(N-p)$ if $N>p, \tilde{p}=\infty$ otherwise. 
Let us assume

$$
\begin{gathered}
W=\left(W_{0}^{1, p}(\Omega)\right)^{n}(n \geq 1) \quad \text { with }\|v\|=\left(\sum_{\ell=1}^{n} \int_{\Omega}\left|\nabla v_{\ell}\right|^{p} d x\right)^{1 / p} \forall v=\left(v_{1}, \ldots, v_{n}\right) \in W \\
B_{\ell}\left(v_{\ell}\right)=p^{-1} \int_{\Omega} b_{\ell}\left|v_{\ell}\right|^{p} d x \quad \forall v_{\ell} \in W_{0}^{1, p}(\Omega) \quad \text { where } b_{\ell} \in L^{\infty}(\Omega) \backslash\{0\}, b_{\ell} \geq 0, \widehat{B}_{\ell} \equiv 0 .
\end{gathered}
$$

Moreover we consider the functionals $A$ (as in $\left(i_{11}\right)$ ) such that

$$
\exists \widetilde{c}>0: A(v) \geq p^{-1} \tilde{c}\|v\|^{p} \quad \forall v \in W .
$$

Let us use the notation $H_{\lambda}\left(S_{\lambda}\right.$ and $V_{\lambda}^{-}$, resp.) instead of $H_{\lambda \mu}\left(S_{\lambda \mu}\right.$ and $V_{\lambda \mu^{\prime}}^{-}$resp.).

As $\ell=1, \ldots, n$ let $\lambda_{\ell}^{*}$ and $u_{\ell}^{*}$, respectively, the first eigenvalue and the first eigenfunction of the problem:

$$
u_{\ell} \in W_{0}^{1, p}(\Omega):-\tilde{c} \operatorname{div}\left(\left|\nabla u_{\ell}\right|^{p-2} \nabla u_{\ell}\right)=\theta b_{\ell}\left|u_{\ell}\right|^{p-2} u_{\ell} \quad \text { in } \Omega
$$

Let us remember that [4]

$$
\begin{aligned}
& u_{\ell}^{*} \in C^{1, \alpha_{\ell}}(\bar{\Omega}) \text { with } 0<\alpha_{\ell}<1, u_{\ell}^{*}>0 \text { in } \Omega ; \\
& \lambda_{\ell}^{*}=\tilde{c} \int_{\Omega}\left|\nabla u_{\ell}^{*}\right|^{p} d x / \int_{\Omega} b_{\ell}\left|u_{\ell}^{*}\right|^{p} d x=\min \left\{\tilde{c} \int_{\Omega}\left|\nabla v_{\ell}\right|^{p} d x / \int_{\Omega} b_{\ell}\left|v_{\ell}\right|^{p} d x: \int_{\Omega} b_{\ell}\left|v_{\ell}\right|^{p} d x>\right. \\
& 0\} ; \\
& \lambda_{\ell}^{*} \text { is simple, that is, each eigenfunction of (2.3) related to } \lambda_{\ell}^{*} \text { is of the type } c_{\ell} u_{\ell}^{*} \text { with } \\
& c_{\ell} \in R \backslash\{0\} ; \\
& \lambda_{\ell}^{*} \text { is isolate, that is, there exists } a>0 \text { such that } \lambda_{\ell}^{*} \text { is the only eigenvalue of (2.3) } \\
& \text { belonging to ]0, } a[\text {. }
\end{aligned}
$$

Remark 2.1. About the results related to problem (2.3), it is sufficient to suppose $b_{\ell} \in L^{\infty}(\Omega)$ and $b_{\ell}^{+}=\max \left\{b_{\ell}, 0\right\} \not \equiv 0$ as $\ell=1, \ldots, n$. This holds also for the results of this section if we limit to consider only the parameters $\lambda_{1}, \ldots, \lambda_{n}$ nonnegative.

Let us start by presenting some sufficient conditions such that $\left(i_{13}\right),\left(i_{14}\right)$, and $\left(i_{15}\right)$ hold. proposition.

Using the variational characterization of $\lambda_{\ell}^{*}$ it is easy to verify the following

Proposition 2.2. If $\lambda_{\ell}<\lambda_{\ell}^{*}$ for all $\ell \in\{1, \ldots, n\}$, then ( $\left.i_{13}\right)$ holds. Consequently, $\left(i_{14}\right)$ holds when $V^{+}\left(D_{m}\right) \neq \emptyset$.

When $\lambda_{\ell} \geq \lambda_{\ell}^{*}$ for some $\ell \in\{1, \ldots, n\}$, it is possible to fulfil ( $\left.i_{14}\right)$ with an additional condition on $D_{m}$. Let $I=\{1, \ldots, n\}$. For any $I^{*} \subseteq I$ let

$$
\begin{aligned}
V^{*}=\{v & =\left(v_{1}, \ldots, v_{n}\right) \in W: v_{\ell} \equiv 0 \text { if } \ell \in I \backslash I^{*}, \\
& \left.v_{\ell}=c_{\ell} u_{\ell}^{*} \text { if } \ell \in I^{*} \text { with } c_{\ell} \in R \text { and } c_{\ell} \neq 0 \text { for some } \ell\right\},
\end{aligned}
$$

and let us suppose

$\left(i_{21}\right)$ There exists $I^{*} \subseteq I: D_{m}(v)<0$ for all $v \in V^{*}$. 
Proposition 2.3. Let $\left(i_{21}\right)$ holds with $I^{*} \neq I$. Let $V^{+}\left(D_{m}\right) \neq \emptyset$. If we fix the parameters set $\left(\lambda_{\ell}\right)_{\ell \in I I I^{*}}$ with $\lambda_{\ell}<\lambda_{\ell^{\prime}}^{*}$, then there exists $\delta^{*}>0$ such that $\left(i_{14}\right)$ also holds for any $\left(\lambda_{\ell}\right)_{\ell \in I^{*}} \in X_{\ell \in I^{*}}\left[\lambda_{\ell}^{*}, \lambda_{\ell}^{*}+\delta^{*}[\right.$.

Proof. Arguing by contradiction, for any $k \in \mathbb{N}$ there exist $\left(\lambda_{\ell}^{k}\right)_{\ell \in I^{*}} \in X_{\ell \in I^{*}}\left[\lambda_{\ell}^{*}, \lambda_{\ell}^{*}+k^{-1}[\right.$ and $v^{k}=\left(v_{1}^{k}, \ldots, v_{n}^{k}\right) \in V^{+}\left(D_{m}\right)$ such that

$$
A\left(v^{k}\right)-p^{-1} \sum_{\ell \in I \backslash I^{*}} \lambda_{\ell} \int_{\Omega} b_{\ell}\left|v_{\ell}\right|^{p} d x-p^{-1} \sum_{\ell \in I^{*}} \lambda_{\ell}^{k} \int_{\Omega} b_{\ell}\left|v_{\ell}^{k}\right|^{p} d x<k^{-1}\left\|v^{k}\right\|^{p}
$$

Set $w^{k}=\left\|v^{k}\right\|^{-1} v^{k}$, we have

$$
\begin{gathered}
D_{m}\left(w^{k}\right)>0, \\
\tilde{c} \sum_{\ell \in I \backslash I^{*}} \int_{\Omega}\left|\nabla w_{\ell}^{k}\right|^{p} d x-\sum_{\ell \in I \backslash I^{*}} \lambda_{\ell} \int_{\Omega} b_{\ell}\left|w_{\ell}^{k}\right|^{p} d x+\tilde{c} \sum_{\ell \in I^{*}} \int_{\Omega}\left|\nabla w_{\ell}^{k}\right|^{p} d x-\sum_{\ell \in I^{*}} \lambda_{\ell}^{k} \int_{\Omega} b_{\ell}\left|w_{\ell}^{k}\right|^{p} d x<p k^{-1},
\end{gathered}
$$

moreover, since $\left\|w^{k}\right\|=1$, there exists $w \in W$ such that (within a subsequence)

$$
w^{k} \longrightarrow w \text { weakly in } W, \quad w^{k} \longrightarrow w \text { strongly in }\left(L^{p}(\Omega)\right)^{n} .
$$

Taking into account that $D_{m}$ is weakly continuous in $W$, from (2.6) as $k \rightarrow+\infty$ we get

$$
\begin{gathered}
D_{m}(w) \geq 0 \\
\sum_{\ell \in I \backslash I^{*}}\left[\tilde{c} \int_{\Omega}\left|\nabla w_{\ell}\right|^{p} d x-\lambda_{\ell} \int_{\Omega} b_{\ell}\left|w_{\ell}\right|^{p} d x\right]+\sum_{\ell \in I^{*}}\left[\tilde{c} \int_{\Omega}\left|\nabla w_{\ell}\right|^{p} d x-\lambda_{\ell}^{*} \int_{\Omega} b_{\ell}\left|w_{\ell}\right|^{p} d x\right] \leq 0
\end{gathered}
$$

Since

$$
\begin{gathered}
w_{\ell} \not \equiv 0 \Longrightarrow \widetilde{c} \int_{\Omega}\left|\nabla w_{\ell}\right|^{p} d x-\lambda_{\ell} \int_{\Omega} b_{\ell}\left|w_{\ell}\right|^{p} d x>0, \\
\tilde{c} \int_{\Omega}\left|\nabla w_{\ell}\right|^{p} d x-\lambda_{\ell}^{*} \int_{\Omega} b_{\ell}\left|w_{\ell}\right|^{p} d x \geq 0
\end{gathered}
$$

from (2.9), we deduce that

$$
w_{\ell} \equiv 0 \quad \forall \ell \in I \backslash I^{*}, \quad \forall \ell \in I^{*} \exists c_{\ell} \in R: w_{\ell}=c_{\ell} u_{\ell}^{*} .
$$

Let us add that $c_{\ell} \neq 0$ for some $\ell \in I^{*}$, since if $c_{\ell}=0$ for all $\ell \in I^{*}$ we have the contradiction $\tilde{c}=\tilde{c} \lim _{k \rightarrow+\infty}\left\|w^{k}\right\|^{p}=0$. Then $w \in V^{*}$, and consequently $D_{m}(w)<0$ from $\left(i_{21}\right)$. This last inequality contradicts (2.8).

In the same way the following propositions can be proved. 
Proposition 2.4. Let $\left(i_{21}\right)$ holds with $I^{*}=I$. Let $V^{+}\left(D_{m}\right) \neq \emptyset$. Then, there exists $\delta^{*}>0$ such that $\left(i_{14}\right)$ also holds for any $\left(\lambda_{\ell}\right)_{\ell \in I} \in X_{\ell \in I}\left[\lambda_{\ell}^{*}, \lambda_{\ell}^{*}+\delta^{*}[\right.$.

Let us pass to $\left(i_{15}\right)$ and suppose

$\left(i_{22}\right)$ there exist $I^{*} \subseteq I$ and $m_{1} \in\{1, \ldots, m\}$ such that $D_{m_{1}}(v)<0$ and $A(v)=$ $\widetilde{c} p^{-1} \sum_{\ell \in I^{*}} \int_{\Omega}\left|\nabla v_{\ell}\right|^{p} d x$ for any $v \in V^{*}$.

Proposition 2.5. If $\left(i_{22}\right)$ holds with $I^{*} \neq I$, then

$$
V_{\lambda}^{-} \cap S\left(D_{m_{1}}\right) \neq \emptyset \quad \forall\left(\lambda_{\ell}\right)_{\ell \in I} \quad \text { with }\left(\lambda_{\ell}\right)_{\ell \in I^{*}} \in \underset{\ell \in I^{*}}{X}\left[\lambda_{\ell}^{*},+\infty\left[\backslash\left\{\left(\lambda_{\ell}^{*}\right)_{\ell \in I^{*}}\right\}\right.\right.
$$

Moreover, if we fix the parameters set $\left(\lambda_{\ell}\right)_{\ell \in I \backslash I^{*}}$ with $\lambda_{\ell}<\lambda_{\ell}^{*}$, then there exists $\delta^{*}>0$ such that

$$
V_{\lambda}^{-} \cap S\left(D_{m_{1}}\right) \text { is bounded in } W \quad \forall\left(\lambda_{\ell}\right)_{\ell \in I^{*}} \in \underset{\ell \in I^{*}}{X}\left[\lambda_{\ell}^{*}, \lambda_{\ell}^{*}+\delta^{*}\left[\backslash\left\{\left(\lambda_{\ell}^{*}\right)_{\ell \in I^{*}}\right\}\right.\right.
$$

Proof. Let us prove (2.12). Let $v \in V^{*}$ with $v_{\ell}=u_{\ell}^{*}$ if $\ell \in I^{*}$, then $D_{m_{1}}(v)<0$. Let $w=$ $\left|D_{m_{1}}(v)\right|^{-1 \backslash q_{m_{1}}} v$, we have

$$
\begin{gathered}
D_{m_{1}}(w)=\left|D_{m_{1}}(v)\right|^{-1} D_{m_{1}}(v)=-1 \\
H_{\lambda}(w)=p^{-1} \sum_{\ell \in I^{*}}\left[\tilde{c} \int_{\Omega}\left|\nabla w_{\ell}\right|^{p} d x-\lambda_{\ell} \int_{\Omega} b_{\ell}\left|w_{\ell}\right|^{p} d x\right]<0 .
\end{gathered}
$$

Let us prove (2.13). Arguing by contradiction, for any $k \in \mathbb{N}$ there exist $\left(\lambda_{\ell}^{k}\right)_{\ell \in I^{*}} \in$ $X_{\ell \in I^{*}}\left[\lambda_{\ell}^{*}, \lambda_{\ell}^{*}+k^{-1}\left[\right.\right.$ with $\left(\lambda_{\ell}^{k}\right)_{\ell \in I^{*}} \neq\left(\lambda_{\ell}^{*}\right)_{\ell \in I^{*}}$ and $\left(v^{k, h}\right)_{h \in \mathbb{N}} \subseteq V_{\lambda^{k}}^{-} \cap S\left(D_{m_{1}}\right)$, where $\lambda_{\ell}^{k}=\lambda_{\ell}$ if $\ell \in I \backslash I^{*}$, such that

$$
\sup _{h \in \mathbb{N}}\left\|v^{k, h}\right\|=+\infty
$$

Relation (2.15) implies that there exists $\left(h_{k}\right)_{k \in \mathbb{N}} \subseteq \mathbb{N}$ strictly increasing such that

$$
\delta_{k}=\left\|v^{k, h_{k}}\right\| \longrightarrow+\infty \quad \text { as } k \longrightarrow+\infty
$$

Let $w^{k}=\delta_{k}^{-1} v^{k, h_{k}}$, we have

$$
\begin{gathered}
\sum_{\ell \in I \backslash I^{*}}\left[\tilde{c} \int_{\Omega}\left|\nabla w_{\ell}^{k}\right|^{p} d x-\lambda_{\ell} \int_{\Omega} b_{\ell}\left|w_{\ell}^{k}\right|^{p} d x\right]+\sum_{\ell \in I^{*}}\left[\tilde{c} \int_{\Omega}\left|\nabla w_{\ell}^{k}\right|^{p} d x-\lambda_{\ell}^{k} \int_{\Omega} b_{\ell}\left|w^{k}\right|^{p} d x\right]<0, \\
D_{m_{1}}\left(w^{k}\right)=-\delta_{k}^{-q_{m_{1}}},
\end{gathered}
$$

$\exists w \in W:\left(\right.$ within a subsequence) $w^{k} \longrightarrow w$ weakly in $W, w^{k} \longrightarrow w$ strongly in $\left(L^{p}(\Omega)\right)^{n}$. 
Then, as $k \rightarrow+\infty$ we get

$$
\begin{gathered}
\sum_{\ell \in I \backslash I^{*}}\left[\tilde{c} \int_{\Omega}\left|\nabla w_{\ell}\right|^{p} d x-\lambda_{\ell} \int_{\Omega} b_{\ell}\left|w_{\ell}\right|^{p} d x\right]+\sum_{\ell \in I^{*}}\left[\tilde{c} \int_{\Omega}\left|\nabla w_{\ell}\right|^{p} d x-\lambda_{\ell}^{*} \int_{\Omega} b_{\ell}\left|w_{\ell}\right|^{p} d x\right] \leq 0 \\
D_{m_{1}}(w)=0
\end{gathered}
$$

From (2.18), we get that $w \in V^{*}$. Then since $\left(i_{22}\right)$ inequality $D_{m_{1}}(w)<0$ holds, which contradicts (2.19).

Proposition 2.6. If $\left(i_{22}\right)$ holds with $I^{*}=I$, then

$$
\begin{gathered}
V_{\lambda}^{-} \cap S\left(D_{m_{1}}\right) \neq \emptyset \quad \forall \lambda=\left(\lambda_{\ell}\right)_{\ell \in I} \in \underset{\ell \in I}{X}\left[\lambda_{\ell}^{*},+\infty\left[\backslash\left\{\left(\lambda_{\ell}^{*}\right)_{\ell \in I}\right\},\right.\right. \\
\exists \delta^{*}>0: V_{\lambda}^{-} \cap S\left(D_{m_{1}}\right) \text { is bounded in } W \quad \forall \lambda=\left(\lambda_{\ell}\right)_{\ell \in I} \in \underset{\ell \in I}{X}\left[\lambda_{\ell}^{*}, \lambda_{\ell}^{*}+\delta^{*}\left[\backslash\left\{\left(\lambda_{\ell}^{*}\right)_{\ell \in I}\right\} .\right.\right.
\end{gathered}
$$

The proof as in Proposition 2.5.

Remark 2.7. The applications we now show, except the first one, deal with systems with $n>1$ equations. We consider the functionals $A$ with $\tilde{c}=1$, and we suppose $b_{\ell} \in L^{\infty}(\Omega) \backslash\{0\}, b_{\ell} \geq 0$. Application 2.8. Let $n=1$. Let us consider the problem

$$
-\operatorname{div}\left(|\nabla u|^{p-2} \nabla u\right)=\lambda_{1} b_{1}|u|^{p-2} u+\sum_{j=1}^{m} d_{j}|u|^{q_{j}-2} u \quad \text { in } \Omega, \quad u=0 \text { on } \partial \Omega,
$$

where

$$
\begin{gathered}
p<q_{1}<\tilde{p}, \quad d_{1} \in L^{\infty}(\Omega) \backslash\{0\} \quad \text { if } m=1, \\
p<q_{1}<\cdots<q_{m}<\tilde{p}, \quad d_{j} \in L^{\infty}(\Omega) \backslash\{0\} \quad \text { as } j=1, \ldots, m, \\
d_{j} \leq 0 \quad \text { as } j=1, \ldots, m-1 \quad \text { if } m>1 .
\end{gathered}
$$

Evidently

$$
A(v)=p^{-1} \int_{\Omega}|\nabla v|^{p} d x, \quad D_{j}(v)=q_{j}^{-1} \int_{\Omega} d_{j}|v|^{q_{j}} d x \quad \forall v \in W .
$$

Let us advance the conditions:

$$
\begin{aligned}
& d_{m}^{+} \not \equiv 0\left(\Longrightarrow V^{+}\left(D_{m}\right) \neq \emptyset\right) \\
& \int_{\Omega} d_{m}\left(u_{1}^{*}\right)^{q_{m}} d x<0 \quad\left(\Longrightarrow D_{m}\left(c_{1} u_{1}^{*}\right)<0 \forall c_{1} \in R \backslash\{0\}\right) .
\end{aligned}
$$


Let us note that (Propositions 2.2, 2.4, and 2.6)

$$
\begin{gathered}
(2.24) \Longrightarrow\left(\left(i_{14}\right) \text { holds if } \lambda_{1}<\lambda_{1}^{*}\right), \\
(2.24) \text { and }(2.25) \Longrightarrow\left(\exists \delta_{1}^{*}>0:\left(i_{14}\right) \text { holds if } \lambda_{1}<\lambda_{1}^{*}+\delta_{1}^{*}\right), \\
(2.25) \Longrightarrow\left(\exists \delta_{2}^{*}>0:\left(i_{15}\right) \text { holds if } \lambda_{1} \in\right] \lambda_{1}^{*}, \lambda_{1}^{*}+\delta_{2}^{*}[) .
\end{gathered}
$$

Proposition 2.9 (see [1], Theorems 2.1, 2.2, 4.1, and 4.2; Remarks 2.1, 2.3, 4.1, and 4.4; Proposition A.3; $[5,6])$. Under assumptions (2.22) we have:

(i) When (2.24) holds, with $\lambda_{1}<\lambda_{1}^{*}$ [resp. (2.24) and (2.25) hold, with $\lambda_{1}<\lambda_{1}^{*}+\delta_{1}^{*}$ ] problem (2.21) has at least two weak solutions $u^{0}$ and $-u^{0}\left(u^{0}=\tau^{0} v^{0}, \tau^{0}=\right.$ const. $>0, v^{0} \in$ $\left.S_{\lambda_{1}} \cap V^{+}\left(D_{m}\right)\right)$, and it results in $u^{0} \in L^{\infty}(\Omega) \cap C_{\ell \circ c}^{1, \alpha^{0}}(\Omega), u^{0}>0$;

(ii) When (2.25) holds, with $\left.\lambda_{1} \in\right] \lambda_{1}^{*}, \lambda_{1}^{*}+\delta_{2}^{*}[$ problem (2.21) has at least two weak solutions $\bar{u}$ and $-\bar{u}\left(\bar{u}=\bar{\tau} \bar{v}, \bar{\tau}=\right.$ const. $\left.>0, \bar{v} \in V_{\lambda_{1}}^{-} \cap S\left(D_{m}\right)\right)$, and it results in $\bar{u} \in L^{\infty}(\Omega) \cap$ $C_{\ell \circ C}^{1, \bar{\alpha}}(\Omega), \bar{u}>0$.

Consequently, when (2.24) and (2.25) hold, with $\left.\lambda_{1} \in\right] \lambda_{1}^{*}, \lambda_{1}^{*}+\min \left\{\delta_{1}^{*}, \delta_{2}^{*}\right\}[$ problem (2.21) has at least four different weak solutions.

Remark 2.10. Our results include the ones of Drábek and Pohozaev [2] when $m=1$.

Application 2.11. Let us consider the system:

$$
\begin{array}{r}
-\operatorname{div}\left(\left|\nabla u_{i}\right|^{p-2} \nabla u_{i}\right)=\lambda_{i} b_{i}\left|u_{i}\right|^{p-2} u_{i}+\left|\sum_{\ell=1}^{n} d_{\ell} u_{\ell}\right|^{q_{1}-2}\left(\sum_{\ell=1}^{n} d_{\ell} u_{\ell}\right) d_{i}-\widetilde{d}_{i}\left|u_{i}\right|^{q_{1}-2} u_{i} \quad \text { in } \Omega, \\
u_{i}=0 \quad \text { on } \partial \Omega \text { as } i=1, \ldots, n,
\end{array}
$$

where

$$
1<q_{1}<\tilde{p}, \quad q_{1} \neq p, \quad d_{\ell}, \tilde{d}_{\ell} \in L^{\infty}(\Omega), \quad d_{\ell}, \tilde{d}_{\ell}>0 .
$$

System (2.27) is included among Problem (P) with:

$$
\begin{gathered}
A(v)=p^{-1} \sum_{\ell=1}^{n} \int_{\Omega}\left|\nabla v_{\ell}\right|^{p} d x \\
D_{1}(v)=q_{1}^{-1}\left[\int_{\Omega}\left|\sum_{\ell=1}^{n} d_{\ell} v_{\ell}\right|^{q_{1}} d x-\sum_{\ell=1}^{n} \int_{\Omega} \tilde{d}_{\ell}\left|v_{\ell}\right|^{q_{1}} d x\right] \quad \forall v=\left(v_{1}, \ldots, v_{n}\right) \in W .
\end{gathered}
$$


Let us advance the conditions (compatible):

$$
d_{\ell}^{q_{1}}<\tilde{d}_{\ell} \quad \forall \ell \in\{1, \ldots, n\}\left(\Longrightarrow D_{1}\left(0, \ldots, c_{i} u_{i}^{*}, \ldots, 0\right)<0 \text { as } i=1, \ldots, n, c_{i} \in R \backslash\{0\}\right),
$$

there exist $\Omega^{+} \subseteq \Omega$ and a constant $\tilde{c}_{j}>0$ such that $\left|\Omega^{+}\right|_{N}>0$ and

$$
\left(\sum_{\ell \neq j} d_{\ell}+\tilde{c}_{j} d_{j}\right)^{q_{1}}>\sum_{\ell \neq j} \tilde{d}_{\ell}+\widetilde{c}_{j}^{q_{1}} \tilde{d}_{j} \quad \text { in } \Omega^{+}\left(\Longrightarrow V^{+}\left(D_{1}\right) \neq \emptyset\right. \text { (Proposition A.1)). }
$$

Then (Propositions 2.2, 2.3, and 2.5)

$$
(2.31) \Longrightarrow\left(\left(i_{14}\right) \text { holds if } \lambda_{\ell}<\lambda_{\ell}^{*} \forall \ell \in\{1, \ldots, n\}\right),
$$

and set $i \in\{1, \ldots, n\}$

(2.30) and (2.31) $\Longrightarrow\left(\right.$ with $\lambda_{\ell}<\lambda_{\ell}^{*} \forall \ell \neq i \exists \delta_{1}^{*}>0:\left(i_{14}\right)$ holds if $\left.\lambda_{i}<\lambda_{i}^{*}+\delta_{1}^{*}\right)$,

(2.30) $\Longrightarrow\left(\right.$ with $\lambda_{\ell}<\lambda_{\ell}^{*} \forall \ell \neq i \exists \delta_{2}^{*}>0:\left(i_{15}\right)$ holds if $\left.\lambda_{i} \in\right] \lambda_{i}^{*}, \lambda_{i}^{*}+\delta_{2}^{*}[)$.

Taking into account that $D_{1}\left(v_{1}, \ldots, v_{n}\right) \leq D_{1}\left(\left|v_{1}\right|, \ldots,\left|v_{n}\right|\right)$ and $D_{1}(-v)=D_{1}(v)$, from ([1], Theorem 2.1, Remark 2.1, and Theorem 4.1) we get the following proposition.

Proposition 2.12. Under assumptions (2.28) we have:

(i) When (2.31) holds, ((2.30) and (2.31) hold resp.), choosing $\lambda_{1}, \ldots, \lambda_{n}$ as in (2.32) (resp. (2.33)) system (2.27) has at least two weak solutions $u^{0}$ and $-u^{0}$ with $u_{\ell}^{0} \geq 0$ as $\ell=$ $1, \ldots, n\left(u^{0}=\tau^{0} v^{0}, \tau_{0}=\right.$ const. $\left.>0, v^{0} \in S_{\curlywedge} \cap V^{+}\left(D_{1}\right)\right)$;

(ii) When (2.30) holds, choosing $\lambda_{1}, \ldots, \lambda_{n}$ as in (2.34) system (2.27) has at least two weak solutions $\bar{u}$ and $-\bar{u}\left(\bar{u}=\bar{\tau} \bar{v}, \bar{\tau}=\right.$ const. $\left.>0, \bar{v} \in V_{\lambda}^{-} \cap S\left(D_{1}\right)\right)$.

Consequently, when (2.30) and (2.31) hold, with $\lambda_{\ell}<\lambda_{\ell}^{*}$ for all $\ell \neq i$ and $\left.\lambda_{i} \in\right] \lambda_{i}^{*}, \lambda_{i}^{*}+$ $\min \left\{\delta_{1}^{*}, \delta_{2}^{*}\right\}[$ system (2.27) has at least four different weak solutions.

The following proposition is obvious. 
Proposition 2.13. The following relations hold:

$$
\begin{gathered}
u_{i}^{0} \not \equiv 0 \quad \text { as } i=1, \ldots, n, \\
\exists h, k \in\{1, \ldots, n\}: \bar{u}_{h} \not \equiv 0, \bar{u}_{k} \not \equiv 0 .
\end{gathered}
$$

Proposition 2.14. If $p<q_{1}$, then as $i=1, \ldots, n$ :

$$
u_{i}^{0} \in L^{\infty}(\Omega) \cap C_{\ell \circ c}^{1, \alpha_{i}^{0}}(\Omega), \quad u_{i}^{0}>0 .
$$

Proof. It is easy to prove that

$$
\begin{array}{r}
\sum_{i=1}^{n} \int_{\Omega}\left|\nabla u_{i}^{0}\right|^{p-2} \nabla u_{i}^{0} \cdot \nabla v_{i} d x \leq \int_{\Omega} g\left(\sum_{i=1}^{n} u_{i}^{0}\right)^{p-1}\left(\sum_{i=1}^{n} v_{i}\right) d x \\
\forall v=\left(v_{1}, \ldots, v_{n}\right) \in\left(W_{0}^{1, p}(\Omega) \cap L^{\infty}(\Omega)\right)^{n} \quad \text { with } v_{i} \geq 0,
\end{array}
$$

where $g \in L^{q_{1} /\left(q_{1}-p\right)}(\Omega)$. Then (Proposition A.3) $u_{i}^{0} \in L^{\infty}(\Omega)$ and consequently [5] $u_{i}^{0} \in$ $C_{\text {loc }}^{1, \alpha_{i}^{0}}(\Omega)$.

Let us note that $u_{i}^{0}$ is a weak supersolution to the equation:

$$
-\operatorname{div}\left(\left|\nabla u_{i}\right|^{p-2} \nabla u_{i}\right)=\lambda_{i} b_{i}\left|u_{i}\right|^{p-2} u_{i}-\tilde{d}_{i}\left|u_{i}\right|^{q 1}-2 u_{i} \quad \text { in } \Omega
$$

Then, since (2.35), it must be [6] $u_{i}^{0}>0$.

Let us continue the analysis of system (2.27) under the condition:

$$
\left(\sum_{\ell \neq i} d_{\ell}\right)^{q_{1}}<\min \left\{\tilde{d}_{1}, \ldots, \tilde{d}_{n}\right\} \quad \forall i \in\{1, \ldots, n\},
$$

then

$$
D_{1}\left(c_{1} u_{1}^{*}, \ldots, c_{n} u_{n}^{*}\right)<0 \quad \forall\left(c_{1}, \ldots, c_{n}\right) \in R^{n} \backslash\{0\} \quad \text { with } c_{i}=0 \text { for at least one } i \in\{1, \ldots, n\} .
$$

Hence (Proposition 2.5) if $I^{*} \subseteq I$ and $I^{*} \neq I$ :

$$
\text { (2.39) } \Longrightarrow\left(\text { as } \lambda _ { \ell } < \lambda _ { \ell } ^ { * } \forall \ell \in I \backslash I ^ { * } \exists \delta ^ { * } > 0 : ( i _ { 1 5 } ) \text { holds if } ( \lambda _ { \ell } ) _ { \ell \in I ^ { * } } \in \underset { \ell \in I ^ { * } } { X } \left[\lambda_{\ell}^{*}, \lambda_{\ell}^{*}+\delta^{*}\left[\backslash\left(\lambda_{\ell}^{*}\right)_{\ell \in I^{*}}\right)\right.\right. \text {. }
$$

Proposition 2.15. Under assumptions (2.28) and (2.39), choosing $\lambda_{1}, \ldots, \lambda_{n}$ as in (2.41) system (2.27) has at least two weak solutions $\bar{u}$ and $-\bar{u}\left(\bar{u}=\bar{\tau} \bar{v}, \bar{\tau}=\right.$ const. $\left.>0, \bar{v} \in V_{\lambda}^{-} \cap S\left(D_{1}\right)\right)$ with $\bar{u}_{i} \not \equiv 0$ as $i=1, \ldots, n$. 
Proof. Thanks to ([1], Theorem 4.1), there exists $\bar{v} \in V_{\lambda}^{-} \cap S\left(D_{1}\right)$ such that

$$
H_{\lambda}(\bar{v})=\inf \left\{H_{\lambda}(v): v \in V_{\lambda}^{-} \cap S\left(D_{1}\right)\right\}=\underline{e}, \quad \bar{u}=\bar{\tau} \bar{v} \text { is a weak solution of system (2.27), }
$$

where $\bar{\tau}=\left(-p q_{1}^{-1} \underline{e}\right)^{1 /\left(q_{1}-p\right)}$.

Reasoning by contradiction, let, for example, $\bar{u}_{1} \equiv 0$. Since $-1=D_{1}(\bar{v}) \leq D_{1}\left(0,\left|\bar{v}_{2}\right|\right.$, $\left.\ldots,\left|\bar{v}_{n}\right|\right)$ and from $(2.39) D_{1}\left(0,\left|\bar{v}_{2}\right|, \ldots,\left|\bar{v}_{n}\right|\right)<0$, setting $\delta=\left|D_{1}\left(0,\left|\bar{v}_{2}\right|, \ldots,\left|\bar{v}_{n}\right|\right)\right|^{-1 / q_{1}}$ we have

$$
D_{1}\left(0, \delta\left|\bar{v}_{2}\right|, \ldots, \delta\left|\bar{v}_{n}\right|\right)=-1, \quad H_{\lambda}\left(0, \delta\left|\bar{v}_{2}\right|, \ldots, \delta\left|\bar{v}_{n}\right|\right)=\delta^{p} H_{\lambda}(\bar{v}) \leq H_{\lambda}(\bar{v}),
$$

then $H_{\lambda}\left(0, \delta\left|\bar{v}_{2}\right|, \ldots, \delta\left|\bar{v}_{n}\right|\right)=H_{\lambda}(\bar{v})$. This implies that ([1], see the proof of Theorem 4.1) $\left(0, \bar{\tau} \delta\left|\bar{v}_{2}\right|, \ldots, \bar{\tau} \delta\left|\bar{v}_{n}\right|\right)$ is a weak solution of system $(2.27)$. Then $\left(\sum_{\ell=2}^{n} d_{\ell}\left|\bar{v}_{\ell}\right|\right)^{q_{1}-1} \equiv 0$ from which $\bar{u}_{\ell} \equiv 0$ too as $\ell=2, \ldots, n$.

Condition (2.39) holds in particular when

$$
\left(\sum_{\ell=1}^{n} d_{\ell}\right)^{q_{1}}<\min \left\{\tilde{d}_{1}, \ldots, \tilde{d}_{n}\right\}
$$

Proposition 2.16. Replacing in Proposition 2.15 (2.39) with (2.44), it is right to say that $\bar{u}_{i} \geq 0$ and $\bar{u}_{i} \equiv 0$ as $i=1, \ldots, n$. Consequently, if $p<q_{1}$

$$
\bar{u}_{i} \in L^{\infty}(\Omega) \cap C_{\ell \circ C}^{1, \bar{a}_{i}}(\Omega), \quad \bar{u}_{i}>0 \text { as } i=1, \ldots, n .
$$

Proof. Set $\delta=\left|D_{1}\left(\left|\bar{v}_{1}\right|, \ldots,\left|\bar{v}_{n}\right|\right)\right|^{-1 / q_{1}}$, as in Proposition $2.15\left(\bar{\tau} \delta\left|\bar{v}_{1}\right|, \ldots, \bar{\tau} \delta\left|\bar{v}_{n}\right|\right)$ is a weak solution to system (2.27).

Let us add that since $(2.44) \Rightarrow D_{1}\left(c_{1} u_{1}^{*}, \ldots, c_{n} u_{n}^{*}\right)<0$ for all $\left(c_{1}, \ldots, c_{n}\right) \in R^{n} \backslash\{0\}$, there exists (Proposition 2.6) $\delta^{* *}>0$ such that

$$
\left(i_{15}\right) \text { holds if }\left(\lambda_{\ell}\right)_{\ell \in I} \in \underset{\ell=1}{X}\left[\lambda_{\ell}^{*}, \lambda_{\ell}^{*}+\delta^{* *}\left[\backslash\left\{\left(\lambda_{\ell}^{*}\right)_{\ell \in I}\right\} .\right.\right.
$$

Then the existence of $\bar{u}$ is assured also choosing $\lambda_{1}, \ldots, \lambda_{n}$ as in (2.46), and the conclusions of Proposition 2.16 hold.

Application 2.17. Let us set

$$
\begin{gathered}
\lambda_{1}=\cdots=\lambda_{n}=\bar{\lambda}, \quad b_{1}=\cdots=b_{n}=b \quad\left(\text { then } \lambda_{1}^{*}=\cdots=\lambda_{n}^{*}=\lambda^{*}, u_{1}^{*}=\cdots=u_{n}^{*}=u^{*}\right), \\
A(v)=p^{-1} \sum_{\ell=1}^{n} \int_{\Omega}\left|\nabla v_{\ell}\right|^{p} d x, \quad D_{1}(v)=q_{1}^{-1} \int_{\Omega} d_{1}\left(\sum_{\ell=1}^{n}\left|v_{\ell}\right|^{\gamma}\right)^{q_{1} / \gamma} d x, \quad \forall v=\left(v_{1}, \ldots, v_{n}\right) \in W,
\end{gathered}
$$


where

$$
1<\gamma<q_{1}<\tilde{p}, \quad q_{1} \neq p, d_{1} \in L^{\infty}(\Omega) .
$$

Let us consider the system:

$$
\begin{array}{r}
-\operatorname{div}\left(\left|\nabla u_{i}\right|^{p-2} \nabla u_{i}\right)=\bar{\lambda} b\left|u_{i}\right|^{p-2} u_{i}+d_{1}\left(\sum_{\ell=1}^{n}\left|u_{\ell}\right|^{\gamma}\right)^{\left(q_{1} / \gamma\right)-1}\left|u_{i}\right|^{\gamma-2} u_{i} \quad \text { in } \Omega, \\
u_{i}=0 \quad \text { on } \partial \Omega \text { as } i=1, \ldots, n .
\end{array}
$$

We advance the conditions

$$
\begin{gathered}
d_{1}^{+} \not \equiv 0 \quad\left(\Longrightarrow V^{+}\left(D_{1}\right) \neq \emptyset\right), \\
\int_{\Omega} d_{1}\left(u^{*}\right)^{q_{1}} d x<0 \quad\left(\Longrightarrow D_{1}\left(c_{1} u^{*}, \ldots, c_{n} u^{*}\right)<0 \forall\left(c_{1}, \ldots, c_{n}\right) \in R^{n} \backslash\{0\}\right) .
\end{gathered}
$$

Therefore,

$$
\begin{aligned}
& \text { (2.50) } \Longrightarrow\left(\left(i_{14}\right) \text { holds if } \bar{\lambda}<\lambda^{*}\right) \quad \text { (Proposition 2.2) , } \\
& \text { (2.50) and }(2.51) \Longrightarrow\left(\exists \delta_{1}^{*}>0:\left(i_{14}\right) \text { holds if } \bar{\lambda}<\lambda^{*}+\delta_{1}^{*}\right) \quad \text { (Proposition 2.4), } \\
& \text { (2.51) } \Longrightarrow\left(\exists \delta_{2}^{*}>0:\left(i_{15}\right) \text { holds if } \bar{\lambda} \in\right] \lambda^{*}, \lambda^{*}+\delta_{2}^{*}[) \quad \text { (Proposition 2.6). }
\end{aligned}
$$

Then ([1], Theorems 2.1 and 4.1, and Remarks 2.1 and 4.1).

Proposition 2.18. Under assumption (2.48), we have:

(i) When (2.50) holds, ((2.50) and (2.51) hold resp.), if $\bar{\lambda}<\lambda^{*}\left(\right.$ resp. $\left.\bar{\lambda}<\lambda^{*}+\delta_{1}^{*}\right)$ system (2.49) has at least two weak solutions $u^{0}$ and $-u^{0}$ with $u_{\ell}^{0} \geq 0$ as $\ell=1, \ldots, n\left(u^{0}=\right.$ $\tau^{0} v^{0}, \tau_{0}=$ const. $\left.>0, v^{0} \in S_{\lambda} \cap V^{+}\left(D_{1}\right)\right) ;$

(ii) When (2.51) holds, if $\bar{\lambda} \in] \lambda^{*}, \lambda^{*}+\delta_{2}^{*}[$ system (2.49) has at least two weak solutions $\bar{u}$ and $-\bar{u}$ with $\bar{u}_{\ell} \geq 0$ as $\ell=1, \ldots, n\left(\bar{u}=\bar{\tau} \bar{v}, \bar{\tau}=\right.$ const. $\left.>0, \bar{v} \in V_{\lambda}^{-} \cap S\left(D_{1}\right)\right)$.

Consequently, when (2.50) and (2.51) hold, with $\bar{\lambda} \in] \lambda^{*}, \lambda^{*}+\min \left\{\delta_{1}^{*}, \delta_{2}^{*}\right\}[$ system (2.49) has at least four different weak solutions. 2.1 and 4.1$)$

In order to establish some properties of $u^{0}$ and $\bar{u}$ it is useful to recall that ([1], Theorems

$$
\begin{aligned}
& D_{1}\left(v^{0}\right)=\sup \left\{D_{1}(v): v \in S_{\lambda} \cap V^{+}\left(D_{1}\right)\right\}=\bar{e}, \tau^{0}=\left(q_{1} p^{-1} \bar{e}\right)^{1 /\left(p-q_{1}\right)}, \\
& H_{\lambda}(\bar{v})=\inf \left\{H_{\lambda}(v): v \in V_{\lambda}^{-} \cap S\left(D_{1}\right)\right\}=\underline{e}, \quad \bar{\tau}=\left(-p q_{1}^{-1} \underline{e}\right)^{1 /\left(q_{1}-p\right)} .
\end{aligned}
$$


Proposition 2.19. When $p<q_{1}$, we have

$$
u_{i}^{0} \in L^{\infty}(\Omega) \cap C_{\ell \circ C}^{1, \alpha_{i}^{0}}(\Omega)
$$

besides

$$
u_{i}^{0} \not \equiv 0 \quad \forall i \in\{1, \ldots, n\} \text { if } r<p
$$

Proof. The relation $u_{i}^{0} \in L^{\infty}(\Omega)$ comes from Proposition A.3. Then [5] $u_{i}^{0} \in C_{\ell \circ c}^{1, \alpha_{i}^{0}}(\Omega)$.

About (2.56), it is sufficiently (Remark 1.1) to prove that

$$
\left(i_{16}^{h}\right) \text { holds } \forall h \in\{1, \ldots, n\} \text { with } \mathfrak{F}=S_{\curlywedge} \cap V^{+}\left(D_{1}\right)
$$

Let $v=\left(v_{1}, \ldots, v_{n}\right) \in S_{\curlywedge} \cap V^{+}\left(D_{1}\right)$ with $v_{h} \equiv 0$. Since

$$
v \in V^{+}\left(D_{1}\right) \Longrightarrow\left(\exists \text { a compact set } \mathbb{K} \subseteq \Omega:|\mathbb{K}|_{N}>0, d_{1}>0 \text { and } \psi=\sum_{\ell \neq h}\left|v_{\ell}\right|^{\gamma}>0 \text { in } \mathbb{K}\right)
$$

let (Proposition A.1) $\left(\varphi_{\varepsilon}\right)_{0<\varepsilon<\varepsilon_{0}} \subseteq C_{0}^{\infty}(\Omega)$ with $0 \leq \varphi_{\varepsilon} \leq 1$ such that

$$
\varphi_{\varepsilon} \longrightarrow x \text { strongly in } L^{s}(\Omega), \quad \int_{\Omega}\left|\nabla \varphi_{\varepsilon}\right|^{s} d x \longrightarrow+\infty \quad \text { as } \varepsilon \longrightarrow 0^{+} \forall s \in[1,+\infty[,
$$

where $X$ is the characteristic function of $\mathbb{K}$. Set $\varepsilon$ such that

$$
\int_{\Omega} d_{1} \psi^{\left(q_{1} / \gamma\right)-1} \varphi_{\varepsilon}^{\gamma} d x>0, \quad \delta=p^{-1}\left[\int_{\Omega}\left|\nabla \varphi_{\varepsilon}\right|^{p} d x-\bar{\lambda} \int_{\Omega} b \varphi_{\varepsilon}^{p} d x\right]>0
$$

with $v(s)=\left(s^{1 / p} v_{1}, \ldots,(1-s)^{1 / p} \delta^{-1 / p} \varphi_{\varepsilon}, \ldots, s^{1 / p} v_{n}\right)$ it results in

$$
\begin{gathered}
H_{\lambda}(v(s))=\delta^{-1}(1-s) p^{-1}\left[\int_{\Omega}\left|\nabla \varphi_{\varepsilon}\right|^{p} d x-\bar{\lambda} \int_{\Omega} b \varphi_{\varepsilon}^{p} d x\right]+s H_{\lambda}(v)=1 \quad \forall s \in[0,1], \\
\exists s_{0} \in\left[0,1\left[: D_{1}(v(s))>0 \quad \forall s \in\left[s_{0}, 1\right], \quad \lim _{s \rightarrow 1^{-}} \frac{d}{d s} D_{1}(v(s))=-\infty\right.\right.
\end{gathered}
$$

Proposition 2.20. When $p<q_{1}$, we have

$$
\begin{gathered}
\bar{u}_{i} \in L^{\infty}(\Omega) \cap C_{\ell \circ c}^{1, \bar{\alpha}_{i}}(\Omega), \\
\bar{u}_{i}>0 \quad \forall i \in\{1, \ldots, n\} \text { if } p<\gamma .
\end{gathered}
$$


Proof. We can get (2.62) from Proposition A.3 and [5].

About (2.63), it is sufficiently [6] to prove that $\bar{u}_{i} \not \equiv 0$ as $i=1, \ldots, n$. Reasoning by contradiction, let, for example, $\bar{v}_{1} \equiv 0$. We note that

$$
\bar{v} \in V_{\lambda}^{-} \Longrightarrow\left(\exists \ell \in\{2, \ldots, n\}: \int_{\Omega}\left|\nabla \bar{v}_{\ell}\right|^{p} d x-\bar{\lambda} \int_{\Omega} b \bar{v}_{\ell}^{p} d x<0\right)
$$

Let us suppose $\ell=2$ and set $v(s)=\left((1-s)^{1 / \gamma} \bar{v}_{2}, s^{1 / \gamma} \bar{v}_{2}, \bar{v}_{3}, \ldots, \bar{v}_{n}\right)$. Then

$$
\begin{gathered}
D_{1}(v(s))=-1 \quad \forall s \in[0,1], \quad \exists s_{0} \in\left[0,1\left[: H_{\lambda}(v(s))<0 \quad \forall s \in\left[s_{0}, 1\right],\right.\right. \\
\lim _{s \rightarrow 1^{-}} \frac{d}{d s} H_{\lambda}(v(s))=+\infty .
\end{gathered}
$$

Set $s_{1} \in\left[s_{0}, 1\left[\right.\right.$ such that $(d / d s) H_{\lambda}(v(s))>0$ for all $s \in\left[s_{1}, 1[\right.$ and taking into account (2.54), we get the contradiction:

$$
H_{\lambda}(\bar{v}) \leq H_{\lambda}(v(s))<H_{\lambda}(\bar{v}) \quad \forall s \in\left[s_{1}, 1[.\right.
$$

Proposition 2.21. When $r=p<q_{1}$, we allow that as $i=1, \ldots, n$ :

$$
u_{i}^{0}>0, \quad \bar{u}_{i}>0
$$

Proof. The assumption $\gamma=p$ implies that

$$
\begin{gathered}
\forall v=\left(v_{1}, \ldots, v_{n}\right) \in W \backslash\{0\} \quad \text { with } v_{h} \equiv 0 \text { for some } h \in\{1, \ldots, n\}, \\
\exists \widetilde{v}=\left(\widetilde{v}_{1}, \ldots, \widetilde{v}_{n}\right) \in W: \widetilde{v}_{\ell} \not \equiv 0 \quad \text { as } \ell=1, \ldots, n, \quad H_{\lambda}(\widetilde{v})=H_{\lambda}(v), \quad D_{1}(\widetilde{v})=D_{1}(v) .
\end{gathered}
$$

Let, for example, $v_{1} \equiv 0$ and $v_{2} \neq \equiv 0$. Set $\left.s \in\right] 0,1\left[\right.$ and $v_{1}^{1}=(1-s)^{1 / p} v_{2}, v_{2}^{1}=s^{1 / p} v_{2}, v_{\ell}^{1}=v_{\ell}$ as $\ell>2$, with $v^{1}=\left(v_{1}^{1}, \ldots, v_{n}^{1}\right)$, we have

$$
H_{\lambda}\left(v^{1}\right)=H_{\lambda}(v), \quad D_{1}\left(v^{1}\right)=D_{1}(v)
$$

If $v_{3} \equiv 0$, set $v_{1}^{2}=(1-s)^{1 / p} v_{1}^{1}, v_{3}^{2}=s^{1 / p} v_{1}^{1}, v_{\ell}^{2}=v_{\ell}^{1}$ as $\ell \in\{1, \ldots, n\} \backslash\{1,3\}$, with $v^{2}=$ $\left(v_{1}^{2}, \ldots, v_{n}^{2}\right)$, it results in

$$
H_{\lambda}\left(v^{2}\right)=H_{\lambda}(v), \quad D_{1}\left(v^{2}\right)=D_{1}(v)
$$

This method let us to find $\widetilde{v}$. 
Then, if $v_{h}^{0} \equiv 0$ (resp. $\bar{v}_{h} \equiv 0$ ) for some $h \in\{1, \ldots, n\}$, with $\widetilde{v}^{0}$ (resp. $\tilde{\bar{v}}$ ) as in (2.68) we have from $(2.53)(\operatorname{resp} .(2.54)) D_{1}\left(\widetilde{v}^{0}\right)=\bar{e}\left(\operatorname{resp} . H_{\lambda}(\tilde{\bar{v}})=e\right.$ ). Consequently ([1], see the proof of Theorem 2.1 (resp. Theorem 4.1)) $\tilde{u}^{0}=\tau^{0} \widetilde{v}^{0}$ (resp. $\widetilde{\bar{u}}=\bar{\tau} \tilde{\bar{v}}$ ) is a weak solution of system (2.49). Therefore [6] $\tilde{u}_{i}^{0}>0$ (resp. $\widetilde{\bar{u}}_{i}>0$ ) as $i=1, \ldots, n$.

Application 2.22. Let us assume $\lambda_{\ell}, b_{\ell}$, and $A$ as in Application 2.17,

$$
D_{j}(v)=q_{j}^{-1} \int_{\Omega} d_{j}\left(\sum_{\ell=1}^{n}\left|v_{\ell}\right|^{\gamma_{j}}\right)^{q_{j} / \gamma_{j}} d x \quad \forall v=\left(v_{1}, \ldots, v_{n}\right) \in W \quad \text { as } j=1, \ldots, m,
$$

where

$$
\begin{gathered}
p<q_{1}<\cdots<q_{m}<\tilde{p}, \quad 1<\gamma_{j}<q_{j}, \quad d_{m} \in L^{\infty}(\Omega), \\
d_{j} \in L^{\infty}(\Omega) \backslash\{0\}, \quad d_{j} \leq 0 \text { if } j=1, \ldots, m-1 .
\end{gathered}
$$

Let us consider the system:

$$
\begin{gathered}
-\operatorname{div}\left(\left|\nabla u_{i}\right|^{p-2} \nabla u_{i}\right)=\bar{\lambda} b\left|u_{i}\right|^{p-2} u_{i}+\sum_{j=1}^{m} d_{j}\left(\sum_{\ell=1}^{n}\left|u_{\ell}\right|^{\gamma_{j}}\right)^{\left(q_{j} / \gamma_{j}\right)-1}\left|u_{i}\right|^{\gamma_{j}-2} u_{i} \quad \text { in } \Omega, \\
u_{i}=0 \quad \text { on } \partial \Omega \text { as } i=1, \ldots, n,
\end{gathered}
$$

under almost one of the conditions:

$$
d_{m}^{+} \not \equiv 0, \quad \int_{\Omega} d_{m}\left(u^{*}\right)^{q_{m}} d x<0
$$

By using some results ([1], Theorems 2.2 and 4.2, and Remarks 2.3 and 4.4), we can advance a proposition similar to Proposition 2.18 replacing in particular $V^{+}\left(D_{1}\right)$ with $V^{+}\left(D_{m}\right)$ and $S\left(D_{1}\right)$ with $S\left(D_{m}\right)$.

Thanks to Proposition A.3 and a result of [5], for the solutions $u^{0}$ and $\bar{u}$ to system (2.73), we have

$$
u_{i}^{0} \in L^{\infty}(\Omega) \cap C_{\ell \circ \mathrm{c}}^{1, \alpha_{i}^{0}}(\Omega), \quad \bar{u}_{i} \in L^{\infty}(\Omega) \cap C_{\ell \mathrm{oc}}^{1, \bar{\alpha}_{i}}(\Omega)
$$

We continue to analyze the properties of $u^{0}$ and $\bar{u}$. To this aim we recall that ([1], Theorems 2.2 and 4.2), set for each $v \in V^{+}\left(D_{m}\right)$ (resp. $\left.v \in V_{\lambda}^{-} \cap S\left(D_{m}\right)\right) \psi(t, v)=p t^{p-1} H_{\lambda}(v)-$ $\sum_{j=1}^{m} q_{j} t^{q_{j}-1} D_{j}(v)$, we have:

$$
\exists \mid t(v)>0: \psi(t(v), v)=0, \quad \frac{\partial \psi}{\partial t}(t(v), v) \neq 0 .
$$


Besides with $\widetilde{\widetilde{E}}(v)=(t(v))^{p} H_{\lambda}(v)-\sum_{j=1}^{m}(t(v))^{q_{j}} D_{j}(v)$, it results in

$$
\begin{array}{cc}
\tilde{\widetilde{E}}\left(v^{0}\right)=\inf \left\{\tilde{\widetilde{E}}(v): v \in S_{\curlywedge} \cap V^{+}\left(D_{m}\right)\right\}, & \tau^{0}=t\left(v^{0}\right), \\
\widetilde{\widetilde{E}}(\bar{v})=\inf \left\{\widetilde{\widetilde{E}}(v): v \in V_{\lambda}^{-} \cap S\left(D_{m}\right)\right\}, & \bar{\tau}=t(\bar{v}) .
\end{array}
$$

Proposition 2.23. When $\gamma_{m}<p \leq \gamma_{j}$ as $j=1, \ldots, m-1$, then

$$
u_{i}^{0} \not \equiv 0 \quad \forall i \in\{1, \ldots, n\}
$$

Proof. It is sufficiently (Remark 1.1) to prove that

$$
\left(i_{16}^{h}\right) \text { holds } \forall h \in\{1, \ldots, n\} \quad \text { with } \mathfrak{F}=S_{\curlywedge} \cap V^{+}\left(D_{m}\right)
$$

Let $v=\left(v_{1}, \ldots, v_{n}\right) \in S_{\lambda} \cap V^{+}\left(D_{m}\right)$ with $v_{h} \equiv 0$. As in Proposition 2.19, it is possible to find $\bar{v}_{h} \in C_{0}^{\infty}(\Omega) \backslash\{0\}$ such that with $v(s)=\left(s^{1 / p_{v}} v_{1}, \ldots,(1-s)^{1 / p} \bar{v}_{h}, \ldots, s^{1 / p} v_{n}\right)$, it results in

$$
\begin{array}{ll}
H_{\lambda}(v(s))=1 \quad \forall s \in[0,1], \quad D_{m}(v(s))>0 & \forall s \in\left[s_{0}, 1\right]\left(0 \leq s_{0}<1\right), \\
\lim _{s \rightarrow 1^{-}} \frac{d}{d s} D_{j}(v(s)) \in R \quad \text { as } j=1, \ldots, m-1, & \lim _{s \rightarrow 1^{-}} \frac{d}{d s} D_{m}(v(s))=-\infty .
\end{array}
$$

Proposition 2.24. When $p<\gamma_{m} \leq \gamma_{j}$ as $j=1, \ldots, m-1$, then

$$
\bar{u}_{i}>0 \quad \forall i \in\{1, \ldots, n\}
$$

Proof. It is sufficiently [6] to prove that $\bar{u}_{i} \not \equiv 0$ for all $i \in\{1, \ldots, n\}$. Reasoning by contradiction, let, for example, $\bar{v}_{1} \equiv 0$ and $\bar{v}_{2} \not \equiv 0$ such that

$$
\int_{\Omega}\left|\nabla \bar{v}_{2}\right|^{p} d x-\bar{\lambda} \int_{\Omega} b \bar{v}_{2}^{p} d x<0 .
$$

Since

$$
t(\bar{v})>0, \quad \psi(t(\bar{v}), \bar{v})=0, \quad \frac{\partial \psi}{\partial t}(t(\bar{v}), \bar{v}) \neq 0,
$$

there exist an open ball $\widetilde{B}$ of $W$ with centre $\bar{v}$ included in $V_{\lambda}^{-}$and a unique functional $t^{*}(v)$ belongs to $C^{1}(\widetilde{B})$ such that

$$
t^{*}(v)>0, \quad \psi\left(t^{*}(v), v\right)=0 \quad \forall v \in \widetilde{B}
$$


Then, the functional

$$
E^{*}(v)=\left(t^{*}(v)\right)^{p} H_{\lambda}(v)-\sum_{j=1}^{m}\left(t^{*}(v)\right)^{q_{j}} D_{j}(v) \quad \forall v \in \widetilde{B}
$$

belongs to $C^{1}(\widetilde{B})$, and we have

$$
t(v)=t^{*}(v) \quad \forall v \in \widetilde{B} \cap S\left(D_{m}\right)
$$

Then, for (2.78)

$$
E^{*}(\bar{v})=\inf \left\{E^{*}(v): v \in \widetilde{B} \cap S\left(D_{m}\right)\right\}
$$

Now, let us remark that with $v(s)=\left((1-s)^{1 / \gamma_{m}} \bar{v}_{2}, s^{1 / \gamma_{m}} \bar{v}_{2}, \bar{v}_{3}, \ldots, \bar{v}_{n}\right)$, it results in

$$
\begin{gathered}
D_{m}(v(s))=-1 \quad \forall s \in[0,1], \quad \exists s_{0} \in\left[0,1\left[: v(s) \in \widetilde{B} \quad \forall s \in\left[s_{0}, 1\right],\right.\right. \\
\lim _{s \rightarrow 1^{-}} \frac{d}{d s} H_{\lambda}(v(s))=+\infty, \quad \lim _{s \rightarrow 1^{-}} \frac{d}{d s} D_{j}(v(s)) \in R \quad \text { as } j=1, \ldots, m-1 .
\end{gathered}
$$

Then, since

$$
\frac{d}{d s} E^{*}(v(s))=\left(t^{*}(v(s))\right)^{p} \frac{d}{d s} H_{\lambda}(v(s))-\sum_{j=1}^{m}\left(t^{*}(v(s))\right)^{q_{j}} \frac{d}{d s} D_{j}(v(s)) \quad \forall s \in\left[s_{0}, 1[,\right.
$$

we have $\lim _{s \rightarrow 1^{-}}(d / d s) E^{*}(v(s))=+\infty$. Consequently,

$$
\exists s_{1} \in\left[s_{0}, 1\left[: \frac{d}{d s} E^{*}(v(s))>0 \quad \forall s \in\left[s_{1}, 1[\right.\right.\right.
$$

from which we get the contradiction:

$$
E^{*}(\bar{v}) \leq E^{*}(v(s))<E^{*}(\bar{v}) \quad \forall s \in\left[s_{1}, 1[.\right.
$$

Proposition 2.25. When $p=\gamma_{1}=\cdots=\gamma_{m}$, we allow that

$$
u_{i}^{0}>0, \quad \bar{u}_{i}>0 \quad \forall i \in\{1, \ldots, n\}
$$

Proof. We reason as in Proposition 2.21, taking into account (2.77) and (2.78) ([1], see proofs of Theorems 2.2 and 4.2). 
Application 2.26. Let for each $v=\left(v_{1}, \ldots, v_{n}\right) \in W$ :

$$
\begin{gathered}
A(v)=p^{-1} \sum_{\ell=1}^{n} \int_{\Omega}\left|\nabla v_{\ell}\right|^{p} d x, \quad D_{j}(v)=-\prod_{\ell=1}^{n} \int_{\Omega}\left|v_{\ell}\right|^{q_{j \ell}} d x \quad \text { as } j=1, \ldots, m-1(m \geq 2), \\
D_{m}(v)=q_{m}^{-1}\left[\int_{\Omega}\left(\sum_{\ell=1}^{n} d_{\ell}\left|v_{\ell}\right|^{\gamma}\right)^{q_{m} / \gamma} d x-\sum_{\ell=1}^{n} \int_{\Omega} \tilde{d}_{\ell}\left|v_{\ell}\right|^{q_{m}} d x\right],
\end{gathered}
$$

where

$$
\begin{gathered}
1<\gamma<p \leq q_{j \ell}, \quad \sum_{\ell=1}^{n} q_{j \ell}=q_{j}<q_{m}<\tilde{p}, \quad q_{1}<\cdots<q_{m-1}, \\
d_{\ell}, \tilde{d}_{\ell} \in L^{\infty}(\Omega), \quad d_{\ell}, \tilde{d}_{\ell}>0 .
\end{gathered}
$$

Let us consider the system:

$$
\begin{aligned}
&-\operatorname{div}\left(\left|\nabla u_{i}\right|^{p-2} \nabla u_{i}\right)= \lambda_{i} b_{i}\left|u_{i}\right|^{p-2} u_{i}-\sum_{j=1}^{m-1}\left(q_{j i} \prod_{\ell \neq i} \int_{\Omega}\left|u_{\ell}\right|^{q_{j \ell}} d x\right)\left|u_{i}\right|^{q_{i j}-2} u_{i} \\
&+\left(\sum_{\ell=1}^{n} d_{\ell}\left|u_{\ell}\right|^{\gamma}\right)^{\left(q_{m} / \gamma\right)-1} d_{i}\left|u_{i}\right|^{\gamma-2} u_{i}-\tilde{d}_{i}\left|u_{i}\right|^{q_{m}-2} u_{i} \quad \text { in } \Omega, \\
& u_{i}=0 \quad \text { on } \partial \Omega \quad \text { as } i=1, \ldots, n .
\end{aligned}
$$

Let us introduce the conditions:

$$
\begin{gathered}
\exists \Omega^{+} \subseteq \Omega:\left|\Omega^{+}\right|_{N}>0, \quad d_{\bar{\ell}}^{q_{m} / \gamma}>\tilde{d}_{\bar{\ell}} \text { in } \Omega^{+} \text {for some } \bar{\ell} \in\{1, \ldots, n-1\}\left(\Longrightarrow V^{+}\left(D_{m}\right) \neq \emptyset\right), \\
d_{n}^{q_{m} / \gamma}<\tilde{d}_{n} \quad\left(\Longrightarrow D_{m}\left(0, \ldots, 0, c_{n} u_{n}^{*}\right)<0 \forall c_{n} \in R \backslash\{0\}\right) .
\end{gathered}
$$

Then (Propositions 2.2, 2.3 and 2.5)

$$
(2.97) \Longrightarrow\left(\text { with } \lambda_{\ell}<\lambda_{\ell}^{*} \forall \ell \in\{1, \ldots, n\} \text { (i } i_{14}\right) \text { holds) }
$$

(2.97) and (2.98) $\Longrightarrow\left(\right.$ with $\lambda_{\ell}<\lambda_{\ell}^{*} \forall \ell \in\{1, \ldots, n-1\} \exists \delta_{1}^{*}>0:\left(i_{14}\right)$ holds if $\left.\lambda_{n}<\lambda_{n}^{*}+\delta_{1}^{*}\right)$,

$$
\text { (2.98) } \Longrightarrow\left(\text { with } \lambda_{\ell}<\lambda_{\ell}^{*} \forall \ell \in\{1, \ldots, n-1\} \exists \delta_{2}^{*}>0:\left(i_{15}\right) \text { holds if } \lambda_{n} \in\right] \lambda_{n}^{*}, \lambda_{n}^{*}+\delta_{2}^{*}[) \text {. }
$$

Since ([1], Theorems 2.2 and 4.2; Remarks 2.3 and 4.4), we get the following proposition. 
Proposition 2.27. Under assumption (2.95) we have:

(i) When (2.97) holds ((2.97) and (2.98) hold, resp.), set $\lambda_{1}, \ldots, \lambda_{n}$ as in (2.99) (resp. (2.100)) system (2.96) has at least two weak solutions $u^{0}$ and $-u^{0}$ with $u_{\ell}^{0} \geq 0$ as $\ell=1, \ldots, n\left(u^{0}=\right.$ $\tau^{0} v^{0}, \tau^{0}=$ const. $\left.>0, v^{0} \in S_{\lambda} \cap V^{+}\left(D_{m}\right)\right)$;

(ii) When (2.98) holds, set $\lambda_{1}, \ldots, \lambda_{n}$ as in (2.101) system (2.96) has at least two weak solutions $\bar{u}$ and $-\bar{u}$ with $\bar{u}_{\ell} \geq 0$ as $\ell=1, \ldots, n\left(\bar{u}=\bar{\tau} \bar{v}, \bar{\tau}=\right.$ const. $\left.>0, \bar{v} \in V_{\lambda}^{-} \cap S\left(D_{m}\right)\right)$.

Consequently, when (2.97) and (2.98) hold, with $\lambda_{\ell}<\lambda_{\ell}^{*}$ for all $\ell \in\{1, \ldots, n-1\}$ and $\left.\lambda_{n} \in\right] \lambda_{n}^{*}, \lambda_{n}^{*}+\min \left\{\delta_{1}^{*}, \delta_{2}^{*}\right\}[$ system (2.96) has at least four different weak solutions.

We remark that (Proposition A.3, [5]) as $i=1, \ldots, n$ :

$$
u_{i}^{0} \in L^{\infty}(\Omega) \cap C_{\ell \circ c}^{1, \alpha_{i}^{0}}(\Omega), \quad \bar{u}_{i} \in L^{\infty}(\Omega) \cap C_{\ell \circ c}^{1, \bar{\alpha}_{i}}(\Omega) .
$$

Moreover, since $u_{i}^{0}$ (resp. $\bar{u}_{i}$ ) is a weak supersolution of the equation:

$$
-\operatorname{div}\left(\left|\nabla u_{i}\right|^{p-2} \nabla u_{i}\right)=\lambda_{i} b_{i}\left|u_{i}\right|^{p-2} u_{i}-\sum_{j=1}^{m-1} a_{j} q_{j i}\left|u_{i}\right|^{q_{j i}-2} u_{i}-\tilde{d}_{i}\left|u_{i}\right|^{q_{m}-2} u_{i} \quad \text { in } \Omega,
$$

where $a_{j}=\prod_{\ell \neq i} \int_{\Omega}\left(u_{\ell}^{0}\right)^{q_{j \ell}} d x$ (resp. $\left.a_{j}=\prod_{\ell \neq i} \int_{\Omega}\left(\bar{u}_{\ell}\right)^{q_{j \ell}} d x\right)$, we have [6]

$$
u_{i}^{0}>0 \text { if } u_{i}^{0} \not \equiv 0\left[\text { resp. } \bar{u}_{i}>0 \text { if } \bar{u}_{i} \not \equiv 0\right] .
$$

Proposition 2.28. It results in

$$
u_{i}^{0}>0 \quad \text { as } i=1, \ldots, n, \quad \bar{u}_{\bar{\ell}}>0 .
$$

Proof. Since (2.104), we must show that

$$
\begin{array}{cl}
u_{i}^{0} \not \equiv 0 & \text { as } i=1, \ldots, n, \\
& \bar{u}_{\bar{\ell}} \neq 0 .
\end{array}
$$

About (2.106), it is sufficient (Remark 1.1) to prove that

$$
\left(i_{16}^{h}\right) \text { holds } \forall h \in\{1, \ldots, n\} \quad \text { with } \mathfrak{F}=S_{\lambda} \cap V^{+}\left(D_{m}\right) .
$$

Let $v=\left(v_{1}, \ldots, v_{n}\right) \in S_{\lambda} \cap V^{+}\left(D_{m}\right)$ with $v_{h} \equiv 0$. Let $\mathbb{K} \subseteq \Omega$ be a compact set such that

$$
|\mathbb{K}|_{N}>0, \quad \psi=\sum_{\ell \neq h} d_{\ell}\left|v_{\ell}\right|^{\gamma}>0 \quad \text { in } \mathbb{K}
$$


From Proposition A.1, there exists $\varphi \in C_{0}^{\infty}(\Omega)$, with $0 \leq \varphi \leq 1$, such that

$$
\delta=p^{-1}\left[\int_{\Omega}|\nabla \varphi|^{p} d x-\lambda_{h} \int_{\Omega} b_{h} \varphi^{p} d x\right]>0, \quad \int_{\Omega} \psi^{q_{m} / \gamma} d_{h} \varphi^{p} d x>0
$$

Then, with $v(s)=\left(s^{1 / p} v_{1}, \ldots,(1-s)^{1 / p} \delta^{-1 / p} \varphi, \ldots, s^{1 / p} v_{n}\right)$, we have

$$
\begin{gathered}
H_{\lambda}(v(s))=1 \quad \forall s \in[0,1], \quad \exists s_{0} \in\left[0,1\left[: D_{m}(v(s))>0 \quad \forall s \in\left[s_{0}, 1\right],\right.\right. \\
\left.\left.\lim _{s \rightarrow 1^{-}} \frac{d}{d s} D_{j}(v(s)) \in\right]-\infty, 0\right] \quad \text { as } j=1, \ldots, m-1, \quad \lim _{s \rightarrow 1^{-}} \frac{d}{d s} D_{m}(v(s))=-\infty .
\end{gathered}
$$

Let us prove (2.107). We recall that ([1], Theorem 4.2):

$$
\widetilde{\widetilde{E}}(\bar{v})=\inf \left\{\widetilde{\widetilde{E}}(v): v \in V_{\lambda}^{-} \cap S\left(D_{m}\right)\right\}
$$

where $\widetilde{\widetilde{E}}$ as in Application 2.22. Reasoning by contradiction, let $\bar{v}_{\bar{\ell}} \equiv 0$. Then, $\bar{v}_{\ell} \not \equiv 0$ for some $\ell \neq \bar{\ell}$ and consequently from (2.104) $\sum_{\ell \neq \bar{\ell}} d_{\ell}\left(\bar{v}_{\ell}\right)^{\gamma}>0$.

Let $\varphi \in C_{0}^{\infty}(\Omega)$, with $0 \leq \varphi \leq 1$, such that $\int_{\Omega} d_{\bar{\ell}}^{q_{m} / \gamma} \varphi^{q_{m}} d x>\int_{\Omega} \tilde{d}_{\bar{\ell}} \varphi^{q_{m}} d x$. Let us consider the function:

$$
\begin{array}{r}
g(s, \tau)=D_{m}\left(\tau \bar{v}_{1}, \ldots, s \varphi, \ldots, \tau \bar{v}_{n}\right) \\
=q_{m}^{-1}\left[\int_{\Omega}\left(s^{\gamma} d_{\bar{\ell}} \varphi^{\gamma}+\tau^{\gamma} \sum_{\ell \neq \bar{\ell}} d_{\ell}\left(\bar{v}_{\ell}\right)^{\gamma}\right)^{q_{m} / \gamma} d x-s^{q_{m}} \int_{\Omega} \tilde{d}_{\bar{\ell}} \varphi^{q_{m}} d x-\tau^{q_{m}} \sum_{\ell \neq \bar{\ell}} \int_{\Omega} \tilde{d}_{\ell}\left(\bar{v}_{\ell}\right)^{q_{m}} d x\right] \\
\forall s \geq 0, \quad \forall \tau \geq 1 .
\end{array}
$$

Since

$$
\begin{gathered}
g(0,1)=-1, \quad \frac{\partial g}{\partial s}(s, \tau)>0 \quad \forall s>0, \forall \tau \geq 1, \quad g(0, \tau)=-\tau^{q_{m}}<-1 \quad \forall \tau>1, \\
\lim _{s \rightarrow+\infty} g(s, \tau)=+\infty \quad \forall \tau \geq 1,
\end{gathered}
$$

we have

$$
\forall \tau \geq 1 \exists \mid s(\tau) \geq 0(s(1)=0, s(\tau)>0 \text { for } \tau>1): g(s(\tau), \tau)=-1 .
$$

We note that $\lim _{\tau \rightarrow 1^{+}} S(\tau)=0$. In fact, if $\left.\left\{\tau_{n}\right\} \subseteq\right] 1,+\infty\left[\right.$ and $\lim \tau_{n}=1$, being $g\left(s\left(\tau_{n}\right), \tau_{n}\right)=$ $-1,\left\{s\left(\tau_{n}\right)\right\}$ is bounded (else (within a subsequence) $\lim g\left(s\left(\tau_{n}\right), \tau_{n}\right)=+\infty$ ). Then (within a subsequence) $\lim s\left(\tau_{n}\right)=\omega$ with $g(\omega, 1)=0$, from which $\omega=0$. 
We add that $s(\tau)$ belongs to $C^{1}(] 1,+\infty[)$, and its derivative has the form:

$$
\left.s^{\prime}(\tau)=-\frac{1}{(s(\tau))^{\gamma-1}} \tilde{g}(s(\tau), \tau) \quad \forall \tau>1 \text { with } \lim _{\tau \rightarrow 1^{+}} \tilde{g}(s(\tau), \tau) \in\right]-\infty, 0[
$$

Hence, set $v(\tau)=\left(\tau \bar{v}_{1}, \ldots, s(\tau) \varphi, \ldots, \tau \bar{v}_{n}\right)$, it results in

$$
\begin{gathered}
D_{m}(v(\tau))=-1 \quad \forall \tau \geq 1, \quad \lim _{\tau \rightarrow 1^{+}} \frac{d}{d \tau} H_{\lambda}(v(\tau))=p H_{\lambda}(\bar{v})<0, \\
\lim _{\tau \rightarrow 1^{+}} \frac{d}{d \tau} D_{j}(v(\tau))=0 \quad \text { as } j=1, \ldots, m-1 .
\end{gathered}
$$

As in Proposition 2.24, we introduce the open ball $\widetilde{B}$ with centre $\bar{v}$ included in $V_{\lambda}^{-}$and the functionals $t^{*}(v)$ and $E^{*}(v)$ belonging to $C^{1}(\widetilde{B})$. Chosen $\tau_{0}>1$ such that $v(\tau) \in \widetilde{B}$ for all $\tau \in$ $\left[1, \tau_{0}\right]$, we have

$$
\frac{d}{d \tau} E^{*}(v(\tau))=\left(t^{*}(v(\tau))\right)^{p} \frac{d}{d \tau} H_{\lambda}(v(\tau))-\sum_{j=1}^{m-1}\left(t^{*}(v(\tau))\right)^{q_{j}} \frac{d}{d \tau} D_{j}(v(\tau)) \quad \forall \tau \in\left[1, \tau_{0}\right]
$$

and consequently $\lim _{\tau \rightarrow 1^{+}}(d / d \tau) E^{*}(v(\tau))<0$. Then, taking into account (2.112), with $\tau_{1} \in$ ] $\left.1, \tau_{0}\right]$ such that $(d / d \tau) E^{*}(v(\tau))<0$ for all $\left.\left.\tau \in\right] 1, \tau_{1}\right]$, we get the contradiction:

$$
\left.\left.E^{*}(\bar{v}) \leq E^{*}(v(\tau))<E^{*}(\bar{v}) \quad \forall \tau \in\right] 1, \tau_{1}\right]
$$

Proposition 2.29. If $d_{\ell}^{q_{m} / r}>\tilde{d}_{\ell}$ as $\ell=1, \ldots, n-1$, then

$$
\bar{u}_{\ell}>0 \quad \text { as } \ell=1, \ldots, n \text {. }
$$

Proof. In fact,

$$
\begin{gathered}
\bar{u}_{\ell}>0 \quad \text { as } \ell=1, \ldots, n-1 \quad(\text { Proposition 2.23) } \\
\bar{u}_{n} \equiv 0 \Longrightarrow D_{m}(\bar{u})>0 .
\end{gathered}
$$


Application 2.30. Let for each $v=\left(v_{1}, \ldots, v_{n}\right) \in W$ :

$$
\begin{gathered}
A(v)=p^{-1} \int_{\Omega}\left(\sum_{\ell=1}^{n}\left|\nabla v_{\ell}\right|^{\gamma}\right)^{p / \gamma} d x+\prod_{\ell=1}^{n} \int_{\Omega}\left|\nabla v_{\ell}\right|^{p_{\ell}} d x \\
D_{j}(v)=\int_{\Omega} \rho_{j}\left(\prod_{\ell=1}^{n}\left|v_{\ell}\right|^{q_{j \ell}}\right) d x \text { as } j=1, \ldots, m-1, \\
D_{m}(v)=q_{m}^{-1}\left[\int_{\Omega}\left|\sum_{\ell=1}^{n} d_{\ell} v_{\ell}\right|^{q_{m}-1}\left(\sum_{\ell=1}^{n} d_{\ell} v_{\ell}\right) d x-\int_{\Omega} d\left|v_{n}\right|^{q_{m}} d x\right],
\end{gathered}
$$

where

$$
\begin{gathered}
1<\gamma<p, p_{\ell}>1, \quad \sum_{\ell=1}^{n} p_{\ell}=p, \quad q_{j \ell}>1, \quad \sum_{\ell=1}^{n} q_{j \ell}=q_{j}, \quad p<q_{m}, q_{1}<\cdots<q_{m}<\tilde{p}, \\
\rho_{j} \in L^{\infty}(\Omega) \backslash\{0\}, \quad \rho_{j} \leq 0, d_{\ell}, d \in L^{\infty}(\Omega), \quad d_{\ell}(x) \neq 0 \text { a.e. in } \Omega, d>0 .
\end{gathered}
$$

Let as $\ell=1, \ldots, n F_{\ell} \in W^{-1, p^{\prime}}(\Omega)\left(p^{\prime}=p /(p-1)\right)$. Let $\langle\langle F, v\rangle\rangle=\sum_{\ell=1}^{n}\left\langle F_{\ell}, v_{\ell}\right\rangle$ for all $v \in W$. Set $\eta_{i}=0$ as $i=1, \ldots, n-1$ and $\eta_{n}=1$, let us consider the system:

$$
\begin{gathered}
-\operatorname{div}\left(\left[\left(\sum_{\ell=1}^{n}\left|\nabla u_{\ell}\right|^{\gamma}\right)^{(p / \gamma)-1}\left|\nabla u_{i}\right|^{\gamma-2}+p_{i}\left(\prod_{\ell \neq i} \int_{\Omega}\left|\nabla u_{\ell}\right|^{p_{\ell}}\right)\left|\nabla u_{i}\right|^{p_{i}-2}\right] \nabla u_{i}\right) \\
=\lambda_{i} b_{i}\left|u_{i}\right|^{p-2} u_{i}+\sum_{j=1}^{m-1} q_{j i} \rho_{j}\left(\prod_{\ell \neq i}\left|u_{\ell}\right|^{q_{j \ell}}\right)\left|u_{i}\right|^{q_{j i}-2} u_{i} \\
+\left|\sum_{\ell=1}^{n} d_{\ell} u_{\ell}\right|^{q_{m}-1} d_{i}-\eta_{i} d\left|u_{n}\right|^{q_{m}-2} u_{n}+F_{i} \quad \text { in } \Omega, \\
u_{i}=0 \quad \text { on } \partial \Omega \text { as } i=1, \ldots, n,
\end{gathered}
$$

under at least one of the following conditions

$\exists \Omega^{+} \subseteq \Omega:\left|\Omega^{+}\right|_{N}>0, \quad d_{\ell}>0 \quad$ in $\Omega^{+}$for some $\ell \in\{1, \ldots, n-1\}\left(\Longrightarrow V^{+}\left(D_{m}\right) \neq \emptyset\right)$,

$$
\left|d_{n}\right|^{q_{m}}<d \quad\left(\Longrightarrow D_{m}\left(0, \ldots, 0, c_{n} u_{n}^{*}\right)<0 \forall c_{n} \in R \backslash\{0\}\right)
$$

Evidently, about the validity of $\left(i_{14}\right)$ we choose $\lambda_{1}, \ldots, \lambda_{n}$ as in Application 2.26.

Proposition 2.31 (see [1], Theorem 3.2). Under assumptions (2.123), (2.125) ((2.125) and (2.126), resp.), if $F \not \equiv 0$ and $\|F\|_{*}$ is sufficiently small, for $\lambda_{1}, \ldots, \lambda_{n}$ as in (2.99) (resp. (2.100)) system (2.124) has at least one weak solution $\tilde{u}\left(\tilde{u}=\tilde{\tau} \tilde{v}, \tilde{\tau}=\right.$ const. $\left.>0, \tilde{v} \in S_{\lambda} \cap V^{+}\left(D_{m}\right)\right)$. 
Let us note that

$$
\tilde{u}_{h} \not \equiv 0 \text { even if } F_{h} \equiv 0 \text { since }\left(F_{h} \equiv 0, \tilde{u}_{h} \equiv 0\right) \Longrightarrow \sum_{\ell=1}^{n} d_{\ell} \tilde{u}_{\ell} \equiv 0 \Longrightarrow D_{m}(\widetilde{u}) \leq 0
$$

Application 2.32. Let $\lambda_{1}=\cdots=\lambda_{n}=0$, and for each $v=\left(v_{1}, \ldots, v_{n}\right) \in W$ :

$$
\begin{gathered}
A(v)=p^{-1} \int_{\Omega}\left[\sum_{\ell=1}^{n}\left(\left|\nabla v_{\ell}\right|^{\gamma}+a\left|v_{\ell}\right|^{\gamma}\right)\right]^{p / \gamma} d x \\
D_{j}(v)=q_{j}^{-1} \int_{\Omega} d_{j}\left(\sum_{\ell=1}^{n}\left|v_{\ell}\right|^{\gamma_{j}}\right)^{q_{j} / \gamma_{j}} d x \quad \text { as } j=1, \ldots, m, \text { with } m>2,
\end{gathered}
$$

under one of the following assumptions:

$$
\begin{gathered}
a \in L^{\infty}(\Omega), \quad a \geq 0, \quad d_{j} \in L^{\infty}(\Omega) \backslash\{0\} \quad \text { with } d_{1} \leq 0, d_{j} \geq 0 \text { as } j \geq 2, \\
1<\gamma_{j}<\gamma<p<q_{2}<\cdots<q_{m}<\tilde{p} \quad \text { as } j \geq 2, \gamma \leq \gamma_{1}<q_{1}<q_{2} ; \\
a \in L^{\infty}(\Omega), \quad a \geq 0, \quad \text { as } j=1, \ldots, m \quad d_{j} \in L^{\infty}(\Omega) \backslash\{0\}, \quad d_{j} \geq 0, \\
1<\gamma_{j}<\gamma<p<q_{1}<\cdots<q_{m}<\tilde{p} .
\end{gathered}
$$

Set $F$ as in Application 2.30. Let us consider the system:

$$
\begin{aligned}
-\operatorname{div}\left(\left[\sum_{\ell=1}^{n}\left(\left|\nabla u_{\ell}\right|^{\gamma}+a\left|u_{\ell}\right|^{\gamma}\right)\right]^{(p / \gamma)-1}\left|\nabla u_{i}\right|^{\gamma-2} \nabla u_{i}\right) \\
=-\left[\sum_{\ell=1}^{n}\left(\left|\nabla u_{\ell}\right|^{\gamma}+a\left|u_{\ell}\right|^{\gamma}\right)\right]^{(p / \gamma)-1} a\left|u_{i}\right|^{\gamma-2} u_{i}+\sum_{j=1}^{m} d_{j}\left(\sum_{\ell=1}^{n}\left|u_{\ell}\right|^{\gamma_{j}}\right)^{\left(q_{j} / \gamma_{j}\right)-1}\left|u_{i}\right|^{\gamma_{j}-2} u_{i}+F_{i} \quad \text { in } \Omega, \\
u_{i}=0 \quad \text { on } \partial \Omega \quad \text { as } i=1, \ldots, n .
\end{aligned}
$$

Let us verify that

(2.129) [resp. (2.130)] $\Longrightarrow\left(\left(i_{16}^{h}\right)\right.$ holds $\forall h \in\{1, \ldots, n\}$ with $\mathfrak{F}=S_{\lambda} \cap V^{+}\left(D_{2}, \ldots, D_{m}\right)$ $\left[\right.$ resp. $\left.\left.\mathfrak{F}=S_{\lambda} \cap V^{+}\left(D_{1}, \ldots, D_{m}\right)\right]\right)$. 
Let $v=\left(v_{1}, \ldots, v_{n}\right) \in \mathfrak{F}$ with, for example, $v_{1} \equiv 0$. Let $j_{0} \in\{2, \ldots, m\}$ (resp. $j_{0} \in$ $\{1, \ldots, m\})$ and $\ell_{0} \in\{2, \ldots, m\}$ such that $d_{j_{0}} v_{\ell_{0}} \not \equiv 0$. Let us suppose $\ell_{0}=2$ and set $v(s)=$ $\left((1-s)^{1 / \gamma} v_{2}, s^{1 / r} v_{2}, v_{3}, \ldots, v_{n}\right)$. Then,

$$
\begin{gathered}
A(v(s))=1 \quad \forall s \in[0,1], \quad \exists s_{0} \in\left[0,1\left[: D_{j_{0}}(v(s))>0 \quad \forall s \in\left[s_{0}, 1\right],\right.\right. \\
\lim _{s \rightarrow 1^{-}} \frac{d}{d s} D_{j_{0}}(v(s))=-\infty, \quad \lim _{s \rightarrow 1^{\prime}} \frac{d}{d s} D_{j}(v(s))<+\infty \quad \text { as } j \neq j_{0} .
\end{gathered}
$$

Proposition 2.33. Under assumption (2.129) (resp. (2.130)), system (2.131) with $F \equiv 0$ has at least two weak solutions $u^{0}$ and $-u^{0}$, and we have as $i=1, \ldots, n$ :

$$
u_{i}^{0} \in L^{\infty}(\Omega), \quad u_{i}^{0} \geq 0, u_{i}^{0} \not \equiv
$$

Consequently,

$$
a \equiv 0 \Longrightarrow u_{i}^{0} \in C_{\ell o c}^{1, \alpha_{i}^{0}}(\Omega), \quad a \equiv 0 \text { and (2.129) holds with } p \leq \gamma_{1}[\text { resp. (2.130) holds }] \Longrightarrow u_{i}^{0}>0
$$

Proof. The statement is due to ([1], Theorem 2.2, Remark 2.3), [5], Proposition A.3, [6].

Proposition 2.34 (see [1], Theorems 3.1, 3.2). Under assumption (2.129) (resp. (2.130)), system (2.131) with $F \not \equiv 0$ and $\|F\|_{*}$ sufficiently small has at least two different weak solutions $u^{1}$ and $u^{2}\left(u^{i}=\tau^{i} v^{i}, \tau^{i}=\right.$ const. $>0, v^{1} \in V^{+}(F) \cap S_{\lambda}, v^{2} \in S_{\lambda} \cap V^{+}\left(D_{2}, \ldots, D_{m}\right)$ [resp. $v^{2} \in$ $\left.S_{\curlywedge} \cap V^{+}\left(D_{1}, \ldots, D_{m}\right)\right]$ ), and we have $u_{h}^{2} \not \equiv 0$ even if $F_{h} \equiv 0$.

Remark 2.35. If $\bigcup_{j=2}^{m}\left\{x \in \Omega: d_{j}(x)>0\right\}\left[\right.$ resp. $\left.\bigcup_{j=1}^{m}\left\{x \in \Omega: d_{j}(x)>0\right\}\right]=\Omega$ (within a set with measure equal to zero), with the same reasoning used about (2.132), we get that

$$
\left(i_{16}^{h}\right) \text { holds } \forall h \in\{1, \ldots, n\} \quad \text { with } \mathfrak{F}=V^{+}(F) \cap S_{\lambda}
$$

hence, $u_{h}^{1} \not \equiv 0$ even if $F_{h} \equiv 0$.

\section{Neumann Problems}

Let $\Omega \subseteq R^{N}$ be an open, bounded, and connected $C^{0,1}$ set. Let $|\cdot|_{N}, p$ and $\tilde{p}$ as in Section 2, $\sigma$ the measure on $\partial \Omega, v$ the outward unit normal to $\partial \Omega, \hat{p}=(N-1) p /(N-p)$ if $p<N, \widehat{p}=\infty$ if 
$p \geq N$. Let us assume

$$
\begin{aligned}
W=\left(W^{1, p}(\Omega)\right)^{n}(n \geq 1) \quad \text { with }\|v\|=\left(\sum_{\ell=1}^{n} \int_{\Omega}\left[\left|\nabla v_{\ell}\right|^{p}+\left|v_{\ell}\right|^{p}\right] d x\right)^{1 / p} \quad \forall v=\left(v_{1}, \ldots, v_{n}\right) \in W, \\
B_{\ell}\left(v_{\ell}\right)=p^{-1} \int_{\Omega} b_{\ell}\left|v_{\ell}\right|^{p} d x \quad \forall v_{\ell} \in W^{1, p}(\Omega), \quad \text { where } b_{\ell} \in L^{\infty}(\Omega) \backslash\{0\} \\
\widehat{B}_{\ell}\left(v_{\ell}\right)=p^{-1} \int_{\partial \Omega} \widehat{b}_{\ell}\left|v_{\ell}\right|^{p} d \sigma \quad \forall v_{\ell} \in W^{1, p}(\Omega), \quad \text { where } \widehat{b}_{\ell} \in L^{\infty}(\partial \Omega) \backslash\{0\} .
\end{aligned}
$$

We note that for each $v_{\ell} \in W^{1, p}(\Omega)$ we set $\gamma_{0}\left(v_{\ell}\right)=v_{\ell}$ where $\gamma_{0}$ is the trace operator from $W^{1, p}(\Omega)$ into $W^{1-(1 / p), p}(\partial \Omega)$. Morever we consider the functionals $A$ (as in $\left(i_{11}\right)$ ) such that

$$
\exists \tilde{c}>0: A(v) \geq p^{-1} \tilde{c} \sum_{\ell=1}^{n} \int_{\Omega}\left|\nabla v_{\ell}\right|^{p} d x \quad \forall v \in W
$$

It is easy to verify the following.

Proposition 3.1. Let $b_{\ell}, \widehat{b}_{\ell} \geq 0$ as $\ell=1, \ldots, n$. Then,

$$
\left(i_{13}\right) \text { holds if } \lambda_{\ell}, \mu_{\ell} \leq 0, \quad \lambda_{\ell}+\mu_{\ell}<0 \text { as } \ell=1, \ldots, n \text {. }
$$

Let us set $I=\{1, \ldots, n\}$ and for each $I^{*} \subseteq I$

$$
C^{*}=\left\{c=\left(c_{1}, \ldots, c_{n}\right) \in R^{n}: c_{\ell}=0 \text { if } \ell \in I \backslash I^{*}, c_{\ell} \neq 0 \text { for some } \ell \in I^{*}\right\}
$$

Let us introduce the conditions:

$\left(i_{31}\right)$ there exists $I^{*} \subseteq I: D_{m}(c)<0$ for all $c \in C^{*}$;

$\left(i_{32}\right)$ there exist $I^{*} \subseteq I$ and $m_{1} \in\{1, \ldots, m\}: D_{m_{1}}(c)<0$ and $A(c)=0$ for all $c \in C^{*}$.

Proposition 3.2. Let $\left(i_{31}\right)$ holds with $I^{*} \neq I$. Let $V^{+}\left(D_{m}\right) \neq \emptyset$. Let $b_{\ell}, \widehat{b}_{\ell} \geq 0$ as $\ell \in I \backslash I^{*}$. Then with $\lambda_{\ell}, \mu_{\ell} \leq 0$ and $\lambda_{\ell}+\mu_{\ell}<0$ as $\ell \in I \backslash I^{*} \exists \delta^{*}>0:\left(i_{14}\right)$ holds if $\left|\lambda_{\ell}\right|,\left|\mu_{\ell}\right| \leq \delta^{*}$ as $\ell \in I^{*}$.

Proof. Reasoning by contradiction, for each $k \in \mathbb{N}$ there exist $\lambda_{\ell}^{k}, \mu_{\ell}^{k} \in\left[-k^{-1}, k^{-1}\right]$, with $\ell \in I^{*}$, and $v^{k}=\left(v_{1}^{k}, \ldots, v_{n}^{k}\right) \in V^{+}\left(D_{m}\right)$ such that

$$
\begin{aligned}
\left\|v^{k}\right\|^{p}>k\{ & A\left(v^{k}\right)-\sum_{\ell \in I \backslash I^{*}} p^{-1}\left[\lambda_{\ell} \int_{\Omega} b_{\ell}\left|v_{\ell}^{k}\right|^{p} d x+\mu_{\ell} \int_{\partial \Omega} \widehat{b}_{\ell}\left|v_{\ell}^{k}\right|^{p} d \sigma\right] \\
& \left.-\sum_{\ell \in I^{*}} p^{-1}\left[\lambda_{\ell}^{k} \int_{\Omega} b_{\ell}\left|v_{\ell}^{k}\right|^{p} d x+\mu_{\ell}^{k} \int_{\partial \Omega} \widehat{b}_{\ell}\left|v_{\ell}^{k}\right|^{p} d \sigma\right]\right\},
\end{aligned}
$$


then, set $w^{k}=\left\|v^{k}\right\|^{-1} v^{k}$, we have

$$
\begin{aligned}
D_{m}\left(w^{k}\right)>0, & p^{-1}\left\{\tilde{c} \sum_{\ell=1}^{n} \int_{\Omega}\left|\nabla w_{\ell}^{k}\right|^{p} d x-\sum_{\ell \in I \backslash I^{*}}\left[\lambda_{\ell} \int_{\Omega} b_{\ell}\left|w_{\ell}^{k}\right|^{p} d x+\mu_{\ell} \int_{\partial \Omega} \widehat{b}_{\ell}\left|w_{\ell}^{k}\right|^{p} d \sigma\right]\right\} \\
& <k^{-1}+\sum_{\ell \in I^{*}} p^{-1}\left[\lambda_{\ell}^{k} \int_{\Omega} b_{\ell}\left|w_{\ell}^{k}\right|^{p} d x+\mu_{\ell}^{k} \int_{\partial \Omega} \widehat{b}_{\ell}\left|w_{\ell}^{k}\right|^{p} d \sigma\right] .
\end{aligned}
$$

Since $\left\|w^{k}\right\|=1$, there exists $w \in W$ such that (within a subsequence)

$w^{k} \longrightarrow w$ weakly in $W, \quad w^{k} \longrightarrow w$ strongly in $\left(L^{p}(\Omega)\right)^{n}, \quad w^{k} \longrightarrow w$ strongly in $\left(L^{p}(\partial \Omega)\right)^{n}$.

Consequently, from (3.6), passing to limit as $k \rightarrow+\infty$, we get

$$
D_{m}(w) \geq 0, \quad \sum_{\ell=1}^{n} \int_{\Omega}\left|\nabla w_{\ell}\right|^{p} d x=0, \quad \sum_{\ell \in I \backslash I^{*}}\left[\lambda_{\ell} \int_{\Omega} b_{\ell}\left|w_{\ell}\right|^{p} d x+\mu_{\ell} \int_{\partial \Omega} \widehat{b}_{\ell}\left|w_{\ell}\right|^{p} d \sigma\right]=0
$$

from which $w=0$, and then the contradiction $0=\lim _{k \rightarrow+\infty}\left\|w^{k}\right\|=1$.

Proposition 3.3. Let $\left(i_{31}\right)$ holds with $I^{*}=I$. Let $V^{+}\left(D_{m}\right) \neq \emptyset$. Then,

$$
\exists \delta^{*}>0:\left(i_{14}\right) \text { holds if }\left|\lambda_{\ell}\right|,\left|\mu_{\ell}\right| \leq \delta^{*} \quad \text { as } \ell=1, \ldots, n \text {. }
$$

The proof as in Proposition 3.2.

Proposition 3.4. Let $\left(i_{32}\right)$ holds with $I^{*} \neq I$. Let $\int_{\Omega} b_{\ell} d x, \int_{\partial \Omega} \widehat{b}_{\ell} d \sigma>0$ as $\ell \in I^{*}$. Then,

$$
V_{\lambda \mu}^{-} \cap S\left(D_{m_{1}}\right) \neq \emptyset \quad \forall\left(\lambda_{\ell}, \mu_{\ell}\right)_{\ell \in I} \text { with } \lambda_{\ell}, \mu_{\ell} \geq 0 \forall \ell \in I^{*}, \quad \lambda_{\ell}+\mu_{\ell}>0 \text { for some } \ell \in I^{*} \text {. }
$$

Moreover, if $b_{\ell}, \widehat{b}_{\ell} \geq 0$ as $\ell \in I \backslash I^{*}$, we have

$$
\begin{aligned}
& \text { with } \lambda_{\ell}, \mu_{\ell} \leq 0 \text { and } \lambda_{\ell}+\mu_{\ell}<0 \text { as } \ell \in I \backslash I^{*} \exists \delta^{*}>0:\left(i_{15}\right) \text { holds } \\
& \text { if } \lambda_{\ell}, \mu_{\ell} \in\left[0, \delta^{*}\right] \forall \ell \in I^{*} \text { and } \lambda_{\ell}+\mu_{\ell}>0 \text { for some } \ell \in I^{*} \text {. }
\end{aligned}
$$

Proof. The first statement is evident. Let us prove the second one. Reasoning by contradiction, for each $k \in \mathbb{N}$ there exist $\lambda_{\ell}^{k}, \mu_{\ell}^{k} \in\left[0, k^{-1}\right]$, with $\ell \in I^{*}$ and $\lambda_{\ell}^{k}+\mu_{\ell}^{k}>0$ for some $\ell \in I^{*}$, and a sequence $\left(v^{k, h}\right)_{h \in \mathbb{N}}$ such that

$$
\left(v^{k, h}\right)_{h \in \mathbb{N}} \subseteq V_{\lambda^{k} \mu^{k}}^{-} \cap S\left(D_{m_{1}}\right) \quad\left(\lambda_{\ell}^{k}=\lambda_{\ell}, \mu_{\ell}^{k}=\mu_{\ell} \text { as } \ell \in I \backslash I^{*}\right), \quad \sup _{h}\left\|v^{k, h}\right\|=+\infty .
$$


Let $\left\{h_{k}\right\} \subseteq \mathbb{N}$ be a strictly increasing sequence such that $\left\|v^{k, h_{k}}\right\| \rightarrow+\infty$ as $k \rightarrow+\infty$. Let $w^{k}=\left\|v^{k, h_{k}}\right\|^{-1} v^{k, h_{k}}$. Then, $D_{m_{1}}\left(w^{k}\right)=-\left\|v^{k, h_{k}}\right\|^{-q_{m_{1}}}$ and

$$
\begin{gathered}
p^{-1}\left\{\tilde{c} \sum_{\ell=1}^{n} \int_{\Omega}\left|\nabla w_{\ell}^{k}\right|^{p} d x-\sum_{\ell \in I I^{*}}\left[\lambda_{\ell} \int_{\Omega} b_{\ell}\left|w_{\ell}^{k}\right|^{p} d x+\mu_{\ell} \int_{\partial \Omega} \widehat{b}_{\ell}\left|w_{\ell}^{k}\right|^{p} d \sigma\right]\right\} \\
<p^{-1} \sum_{\ell \in I^{*}}\left[\lambda_{\ell}^{k} \int_{\Omega} b_{\ell}\left|w_{\ell}^{k}\right|^{p} d x+\mu_{\ell}^{k} \int_{\partial \Omega} \widehat{b}_{\ell}\left|w_{\ell}^{k}\right|^{p} d \sigma\right],
\end{gathered}
$$

moreover, there exists $w \in W$ such that (within a subsequence)

$$
\begin{gathered}
w^{k} \longrightarrow w \text { weakly in } W, \quad w^{k} \longrightarrow w \text { strongly in }\left(L^{p}(\Omega)\right)^{n}, \\
w^{k} \longrightarrow w \text { strongly in }\left(L^{p}(\partial \Omega)\right)^{n} .
\end{gathered}
$$

Consequently,

$$
D_{m_{1}}(w)=0, \quad \sum_{\ell=1}^{n} \int_{\Omega}\left|\nabla w_{\ell}\right|^{p} d x=0, \quad \sum_{\ell \in I \backslash I^{*}}\left[\lambda_{\ell} \int_{\Omega} b_{\ell}\left|w_{\ell}\right|^{p} d x+\mu_{\ell} \int_{\partial \Omega} \widehat{b}_{\ell}\left|w_{\ell}\right|^{p} d \sigma\right]=0,
$$

then $w=0$, and the contradiction $0=\lim _{k \rightarrow+\infty}\left\|w^{k}\right\|=1$.

Proposition 3.5. Let $\left(i_{32}\right)$ holds with $I^{*}=I$. Let $\int_{\Omega} b_{\ell} d x, \int_{\partial \Omega} \widehat{b}_{\ell} d \sigma>0$ as $\ell=1, \ldots, n$. Then,

$$
\begin{gathered}
V_{\lambda \mu}^{-} \cap S\left(D_{m_{1}}\right) \neq \emptyset \quad \text { if } \lambda_{\ell}, \mu_{\ell} \geq 0 \quad \forall \ell \in I \text { and } \lambda_{\ell}+\mu_{\ell}>0 \text { for some } \ell \in I, \\
\exists \delta^{*}>0:\left(i_{15}\right) \text { holds if } \lambda_{\ell}, \mu_{\ell} \in\left[0, \delta^{*}\right] \quad \forall \ell \in I \text { and } \lambda_{\ell}+\mu_{\ell}>0 \text { for some } \ell \in I .
\end{gathered}
$$

The proof as in Proposition 3.4.

Remark 3.6. It is suitable to make some clarifications.

(i) The assumption " $b_{\ell}, \widehat{b}_{\ell} \geq 0$ " (see Propositions 3.1, 3.2, and 3.4) can be replaced by " $b_{\ell}, \widehat{b}_{\ell}$ do not change sign." In this case we can choose $\lambda_{\ell}$ and $\mu_{\ell}$ such that $\lambda_{\ell} b_{\ell} \leq$ $0, \mu_{\ell} \widehat{b}_{\ell} \leq 0$ and $\left|\lambda_{\ell}\right|+\left|\mu_{\ell}\right|>0$.

(ii) The assumption " $\int_{\Omega} b_{\ell} d x, \int_{\partial \Omega} \widehat{b}_{\ell} d \sigma>0$ " (see Propositions 3.4 and 3.5) can be replaced by " $\int_{\Omega} b_{\ell} d x, \int_{\partial \Omega} \widehat{b}_{\ell} d \sigma \neq 0$ ". In this case, we can choose $\lambda_{\ell}$ and $\mu_{\ell}$ such that $\lambda_{\ell} \int_{\Omega} b_{\ell} d x, \mu_{\ell} \int_{\partial \Omega} \widehat{b}_{\ell} d \sigma \geq 0$ and $\left|\lambda_{\ell}\right|+\left|\mu_{\ell}\right|>0$ for some $\ell$, with $\left|\lambda_{\ell}\right|,\left|\mu_{\ell}\right| \leq \delta^{*}$ instead of $\lambda_{\ell}, \mu_{\ell} \in\left[0, \delta^{*}\right]$.

(iii) When for each $\ell \in\{1, \ldots, n\} b_{\ell}, \widehat{b}_{\ell}$ do not change sign, then the conclusion of the Proposition 3.2 [resp. Proposition 3.3] holds even if $\lambda_{\ell} b_{\ell}, \mu_{\ell} \widehat{b}_{\ell} \leq 0$ and $\left|\lambda_{\ell}\right|+\left|\mu_{\ell}\right|>\delta^{*}$ as $\ell \in I^{*}$ (resp. as $\left.\ell=1, \ldots, n\right)$.

In order to simplify the presentation of the applications, we suppose in the next $b_{\ell} \in L^{\infty}(\Omega) \backslash\{0\}$ and $\hat{b}_{\ell} \in L^{\infty}(\partial \Omega) \backslash\{0\}$, while the additional assumptions on $b_{\ell}, \widehat{b}_{\ell}$ and 
the assumptions on $\int_{\Omega} b_{\ell} d x, \int_{\partial \Omega} \widehat{b}_{\ell} d \sigma$ (the same of Propositions 3.1, 3.2, 3.4, and 3.5) will be pointed out case by case.

Passing to the applications (with $n>1$ ), we recall that in [3] Pohozaev and Véron in the case $n=1$ have studied the Neumann problem:

$$
\begin{gathered}
-\operatorname{div}\left(|\nabla u|^{p-2} \nabla u\right)=\lambda b(x)|u|^{p-2} u+c(x)|u|^{s-2} u+a(x)|u|^{q-2} u \quad \text { in } \Omega, \\
|\nabla u|^{p-2} \frac{\partial u}{\partial v}=k(x)|u|^{r-2} u \quad \text { on } \partial \Omega .
\end{gathered}
$$

The existence theorems proved by these authors can be got by using some results of ([1], Theorems 2.1, 2.2, 4.1, and 4.2; Remarks 2.1, 2.3, 4.1, and 4.4), Propositions 3.3 and 3.5.

Application 3.7. Let for each $v=\left(v_{1}, \ldots, v_{n}\right) \in W$ :

$$
A(v)=p^{-1} \sum_{\ell=1}^{n} \int_{\Omega}\left|\nabla v_{\ell}\right|^{p} d x, \quad D_{1}(v)=q_{1}^{-1}\left[\int_{\partial \Omega}\left(\sum_{\ell=1}^{n} d_{\ell}\left|v_{\ell}\right|^{\gamma}\right)^{q_{1} / \gamma} d \sigma-\sum_{\ell=1}^{n} \int_{\partial \Omega} \widehat{d}_{\ell}\left|v_{\ell}\right|^{q_{1}} d \sigma\right],
$$

where

$$
1<\gamma<q_{1}<\widehat{p}, \quad q_{1} \neq p, \quad d_{\ell}, \widehat{d}_{\ell} \in L^{\infty}(\partial \Omega), \quad d_{\ell}, \widehat{d}_{\ell}>0 .
$$

Let us consider the system:

$$
\begin{aligned}
& -\operatorname{div}\left(\left|\nabla u_{i}\right|^{p-2} \nabla u_{i}\right)=\lambda_{i} b_{i}\left|u_{i}\right|^{p-2} u_{i} \quad \text { in } \Omega, \\
\left|\nabla u_{i}\right|^{p-2} \frac{\partial u_{i}}{\partial v}= & \mu_{i} \widehat{b}_{i}\left|u_{i}\right|^{p-2} u_{i}+\left(\sum_{\ell=1}^{n} d_{\ell}\left|u_{\ell}\right|^{\gamma}\right)^{\left(q_{1} / \gamma\right)-1} d_{i}\left|u_{i}\right|^{\gamma-2} u_{i} \\
& -\widehat{d}_{i}\left|u_{i}\right|^{q_{1}-2} u_{i} \quad \text { on } \partial \Omega \text { as } i=1, \ldots, n .
\end{aligned}
$$

Let us introduce the conditions:

$$
\begin{gathered}
\int_{\partial \Omega}\left(\sum_{\ell=1}^{n} d_{\ell}\right)^{q_{1} / \gamma} d \sigma<\int_{\partial \Omega} \widehat{d} d \sigma \quad\left(\widehat{d}=\min \left\{\widehat{d}_{1}, \ldots, \widehat{d}_{n}\right\}\right), \\
\exists \Gamma \subseteq \partial \Omega: \sigma(\Gamma)>0, \quad\left(\sum_{\ell=1}^{n} d_{\ell}\right)^{q_{1} / \gamma}>\sum_{\ell=1}^{n} \widehat{d}_{\ell} \quad \text { on } \Gamma, \\
\int_{\Omega} b_{\ell} d x>0, \quad \int_{\partial \Omega} \hat{b}_{\ell} d \sigma>0 \quad \text { as } \ell=1, \ldots, n .
\end{gathered}
$$


Evidently (3.20) $\Rightarrow D_{1}(c)<0$ for all $c \in R^{n} \backslash\{0\}$. Moreover $(3.21) \Rightarrow V^{+}\left(D_{1}\right) \neq \emptyset$ (Proposition A.2). Hence (Propositions 3.3 and 3.5)

(3.20) and (3.21)

$$
\Longrightarrow\left(\exists \delta_{1}^{*}>0:\left(i_{14}\right) \text { holds if }\left|\lambda_{\ell}\right|,\left|\mu_{\ell}\right| \leq \delta_{1}^{*} \forall \ell \in\{1, \ldots, n\}\right),
$$

(3.20) and (3.22)

$$
\Longrightarrow\left(\exists \delta_{2}^{*}>0\left(i_{15}\right) \text { holds if } \lambda_{\ell}, \mu_{\ell} \in\left[0, \delta_{2}^{*}\right] \forall \ell \in\{1, \ldots, n\}, \lambda_{\ell}+\mu_{\ell}>0 \text { for some } \ell\right) .
$$

Proposition 3.8 (see ([1], Theorems 2.1 and 4.1; Remarks 2.1 and 4.1); Proposition A.4; $[5,6]$ ). Under assumption (3.18), we have:

(i) When (3.20) and (3.21) hold, with $\lambda_{\ell}, \mu_{\ell}$ as in (3.23) system (3.19) has at least two weak solutions $u^{0}$ and $-u^{0}\left(u^{0}=\tau^{0} v^{0}, \tau^{0}=\right.$ const. $\left.>0, v^{0} \in S_{\lambda \mu} \cap V^{+}\left(D_{1}\right)\right)$, and it results in

$$
u_{i}^{0} \in L^{\infty}(\Omega) \cap C_{\ell o c}^{1, \alpha_{i}^{0}}(\Omega), \quad u_{i}^{0} \geq 0 \text { as } i=1, \ldots, n, u_{i}^{0}>0 \text { if } u_{i}^{0} \not \equiv 0
$$

(ii) When (3.20) and (3.22) hold, with $\lambda_{\ell}, \mu_{\ell}$ as in (3.24) system (3.19) has at least two weak solutions $\bar{u}$ and $-\bar{u}\left(\bar{u}=\bar{\tau} \bar{v}, \bar{\tau}=\right.$ const. $\left.>0, \bar{v} \in V_{\lambda \mu}^{-} \cap S\left(D_{1}\right)\right)$, and it results in

$$
\bar{u}_{i} \in L^{\infty}(\Omega) \cap C_{\ell o c}^{1, \bar{\alpha}_{i}}(\Omega), \quad \bar{u}_{i} \geq 0 \text { as } i=1, \ldots, n, \bar{u}_{i}>0 \text { if } \bar{u}_{i} \not \equiv 0 .
$$

Consequently, when (3.20)-(3.22) hold, with $\lambda_{\ell}, \mu_{\ell}$ as in (3.24) and $\min \left\{\delta_{1}^{*}, \delta_{2}^{*}\right\}$ instead of $\delta_{2}^{*}$ system (3.19) has at least four different weak solutions.

Proposition 3.9. If $\gamma<p<q_{1}$, then $u_{i}^{0}>0$ as $i=1, \ldots n$.

Proof. It is sufficient (Remark 1.1) to verify that

$$
\left(i_{16}^{h}\right) \text { holds as } h=1, \ldots, n \text { with } \mathfrak{F}=S_{\lambda \mu} \cap V^{+}\left(D_{1}\right) \text {. }
$$

Let $v=\left(v_{1}, \ldots, v_{n}\right) \in V^{+}\left(D_{1}\right) \cap S_{\lambda \mu}$. Let, for example, $v_{1} \equiv 0$. Since $\int_{\partial \Omega}\left(\sum_{\ell \neq 1} d_{\ell}\left|v_{\ell}\right|^{\gamma}\right)^{q_{1} / \gamma} d \sigma>0$, there exists $\Gamma^{+} \subseteq \partial \Omega$ such that

$$
\sigma\left(\Gamma^{+}\right)>0, \quad \sum_{\ell \neq 1} d_{\ell}\left|v_{\ell}\right|^{\gamma}>0 \quad \text { on } \Gamma^{+} .
$$

Let $\mathbb{K} \subseteq \Omega$ a compact set and $\Omega^{\prime}$ an open set such that

$$
|\mathbb{K}|_{N}>0, \quad \mathbb{K} \subseteq \Omega^{\prime}, \quad \overline{\Omega^{\prime}} \subseteq \Omega .
$$


Since Propositions A.1 and A.2, there exist a compact set $\widehat{\Gamma}^{+} \subseteq \Gamma^{+}$, with $\sigma\left(\widehat{\Gamma}^{+}\right)>0$, and $\left(\varphi_{1 \varepsilon}\right)_{0<\varepsilon<\varepsilon_{0}}\left(\varphi_{2 \varepsilon}\right)_{0<\varepsilon<\varepsilon_{0}} \subseteq C_{0}^{\infty}\left(R^{N}\right)$ such that

$$
\begin{gathered}
0 \leq \varphi_{1 \varepsilon} \leq 1, \quad \operatorname{supp} \varphi_{1 \varepsilon} \subseteq \Omega^{\prime}, \varphi_{1 \varepsilon} \longrightarrow x \text { strongly in } L^{s}(\Omega) \\
\int_{\Omega}\left|\nabla \varphi_{1 \varepsilon}\right|^{s} d x \longrightarrow+\infty \quad \text { as } \varepsilon \longrightarrow 0^{+} \forall s \in[1,+\infty[ \\
0 \leq \varphi_{2 \varepsilon} \leq 1, \quad \operatorname{supp} \varphi_{2 \varepsilon} \subseteq R^{N} \backslash \overline{\Omega^{\prime}}, \varphi_{2 \varepsilon} \longrightarrow \widehat{x} \text { strongly in } L^{s}(\partial \Omega), \\
\int_{\Omega} \varphi_{2 \varepsilon}^{s} d x \longrightarrow 0 \quad \text { as } \varepsilon \longrightarrow 0^{+} \forall s \in[1,+\infty[
\end{gathered}
$$

where $\mathcal{X}($ resp. $\widehat{X})$ is the characteristic function of $\mathbb{K}\left(\right.$ resp. $\left.\widehat{\Gamma}^{+}\right)$. Let us choose $\varepsilon$ such that

$$
\begin{gathered}
\delta=p^{-1}\left[\int_{\Omega}\left|\nabla \varphi_{\varepsilon}\right|^{p} d x-\lambda_{1} \int_{\Omega} b_{1} \varphi_{\varepsilon}^{p} d x-\mu_{1} \int_{\partial \Omega} \widehat{b}_{1} \varphi_{\varepsilon}^{p} d \sigma\right]>0, \\
\int_{\partial \Omega}\left(\sum_{\ell \neq 1} d_{\ell}\left|v_{\ell}\right|^{\gamma}\right)^{\left(q_{1} / \gamma\right)-1} d_{1} \varphi_{\varepsilon}^{\gamma} d \sigma>0 \quad\left(\varphi_{\varepsilon}=\varphi_{1 \varepsilon}+\varphi_{2 \varepsilon}\right),
\end{gathered}
$$

and we set $v(s)=\left((1-s)^{1 / p} \delta^{-1 / p} \varphi_{\varepsilon}, s^{1 / p} v_{2}, \ldots, s^{1 / p} v_{n}\right)$. Then,

$$
\begin{gathered}
H_{\lambda \mu}(v(s))=1 \quad \forall s \in[0,1], \quad \exists s_{0} \in\left[0,1\left[: D_{1}(v(s))>0 \quad \forall s \in\left[s_{0}, 1\right],\right.\right. \\
\lim _{s \rightarrow 1^{-}} \frac{d}{d s} D_{1}(v(s))=-\infty .
\end{gathered}
$$

Proposition 3.10. If

$$
\begin{aligned}
& d_{\ell}^{q_{1} / r}<\widehat{d}_{\ell} \quad \text { as } \ell=1, \ldots, n, \\
& \lambda_{\ell}+\mu_{\ell}>0 \text { as } \ell=1, \ldots, n,
\end{aligned}
$$

then $\bar{u}_{i}>0$ as $i=1, \ldots, n$.

Proof. We recall that ([1], Theorem 4.1)

$$
H_{\lambda \mu}(\bar{v})=\inf \left\{H_{\lambda \mu}(v): v \in V_{\lambda \mu}^{-} \cap S\left(D_{1}\right)\right\}
$$

Reasoning by contradiction let, for example, $\bar{v}_{1} \equiv 0$. As $c_{1}=$ const. $>0$ and $g(s, \tau)=D_{1}\left(s c_{1}, \tau \bar{v}_{2}, \ldots, \tau \bar{v}_{n}\right)=q_{1}^{-1}\left[\int_{\partial \Omega}\left(d_{1} s^{\gamma} c_{1}^{\gamma}+\tau^{\gamma} \sum_{\ell \neq 1} d_{\ell}\left(\bar{v}_{\ell}\right)^{\gamma}\right)^{q_{1} \backslash \gamma} d \sigma-s^{q_{1}} c_{1}^{q_{1}} \int_{\partial \Omega} \widehat{d}_{1} d \sigma-\right.$ $\left.\tau^{q_{1}} \sum_{\ell \neq 1} \int_{\partial \Omega} \widehat{d}_{\ell}\left(\bar{v}_{\ell}\right)^{q_{1}} d \sigma\right]$ for all $s, \tau \geq 0$, we have $g(0, \tau)=-\tau^{q_{1}}>-1$ for all $\left.\tau \in\right] 0,1[$ and since (3.33) $\lim _{s \rightarrow+\infty} g(s, \tau)=-\infty$ for all $\tau \geq 0$. Then for all $\left.\tau \in\right] 0,1[$, it is possible to choose $s(\tau)>0$ such that $g(s(\tau), \tau)=-1$. Let us add that there exist $s_{0}>0$ and $\left.\tau_{0} \in\right] 0,1[$ such that $(\partial g / \partial s)(s, \tau)>0$ for all $(s, \tau) \in] 0, s_{0}[\mathrm{x}] \tau_{0}, 1[$. 
Let now $\left.\left\{\tau_{n}\right\} \subseteq\right] \tau_{0}, 1\left[\right.$ and $\lim \tau_{n}=1$. Since $g\left(s\left(\tau_{n}\right), \tau_{n}\right)=-1,\left\{s\left(\tau_{n}\right)\right\}$ is necessarily bounded. Then (within a subsequence) $\lim s\left(\tau_{n}\right)=\omega \geq s_{0}$. Consequently, from the inequality:

$$
H_{\lambda \mu}(\bar{v}) \leq H_{\lambda \mu}\left(v\left(\tau_{n}\right)\right), \quad \text { where } v\left(\tau_{n}\right)=\left(s\left(\tau_{n}\right) c_{1}, \tau_{n} \bar{v}_{2}, \ldots, \tau_{n} \bar{v}_{n}\right) \in V_{\lambda \mu}^{-} \cap S\left(D_{1}\right)
$$

as $n \rightarrow+\infty$ and from (3.34), we get the contradiction:

$$
H_{\lambda \mu}(\bar{v}) \leq-p^{-1} \omega^{p} c_{1}^{p}\left(\lambda_{1} \int_{\Omega} b_{1} d x+\mu_{1} \int_{\partial \Omega} \widehat{b}_{1} d \sigma\right)+H_{\lambda \mu}(\bar{v})<H_{\lambda \mu}(\bar{v})
$$

Remark 3.11. Let us note that the conditions (3.20), (3.21), and (3.33) are compatible.

Application 3.12. Let for each $v=\left(v_{1}, \ldots, v_{n}\right) \in W$ :

$$
\begin{aligned}
A(v) & =p^{-1}\left[\sum_{\ell=1}^{n-1} \int_{\Omega}\left|\nabla v_{\ell}\right|^{p} d x+\int_{\Omega}\left(\left|\nabla v_{n}\right|^{\gamma}+\int_{\partial \Omega}\left|v_{n}\right|^{\gamma} d \sigma\right)^{p / \gamma} d x\right] \\
D_{1}(v) & =q_{1}^{-1}\left[\sum_{\ell=1}^{n-1} \int_{\Omega} \rho_{\ell}\left|v_{\ell}+v_{n}\right|^{q_{1}-1}\left(v_{\ell}+v_{n}\right) d x-\sum_{\ell=1}^{n} \int_{\partial \Omega} \widehat{d}_{\ell}\left|v_{\ell}\right|^{q_{1}} d \sigma\right]
\end{aligned}
$$

where

$$
1<\gamma<p, \quad 1<q_{1}<\hat{p}, \quad q_{1} \neq p, \quad \rho_{\ell} \in L^{\infty}(\Omega), \quad \rho_{\ell}>0, \quad \widehat{d}_{\ell} \in L^{\infty}(\partial \Omega), \quad \widehat{d}_{\ell}>0 .
$$

Let us consider the system:

$$
\begin{aligned}
& -\operatorname{div}\left(\left|\nabla u_{i}\right|^{p-2} \nabla u_{i}\right)=\lambda_{i} b_{i}\left|u_{i}\right|^{p-2} u_{i}+\rho_{i}\left|u_{i}+u_{n}\right|^{q_{1}-1} \quad \text { in } \Omega \text { as } i=1, \ldots, n-1, \\
& -\operatorname{div}\left[\left(\left|\nabla u_{n}\right|^{\gamma}+\int_{\partial \Omega}\left|u_{n}\right|^{\gamma} d \sigma\right)^{(p / \gamma)-1}\left|\nabla u_{n}\right|^{\gamma-2} \nabla u_{n}\right]=\lambda_{n} b_{n}\left|u_{n}\right|^{p-2} u_{n}+\sum_{\ell=1}^{n-1} \rho_{\ell}\left|u_{\ell}+u_{n}\right|^{q_{1}-1} \text { in } \Omega, \\
& \left|\nabla u_{i}\right|^{p-2} \frac{\partial u_{i}}{\partial v}=\mu_{i} \widehat{b}_{i}\left|u_{i}\right|^{p-2} u_{i}-\widehat{d}_{i}\left|u_{i}\right|^{q_{1}-2} u_{i} \quad \text { on } \partial \Omega \text { as } i=1, \ldots, n-1, \\
& \left(\left|\nabla u_{n}\right|^{\gamma}+\int_{\partial \Omega}\left|u_{n}\right|^{\gamma} d \sigma\right)^{(p / \gamma)-1}\left|\nabla u_{n}\right|^{\gamma-2} \frac{\partial u_{n}}{\partial v} \\
& =\mu_{n} \widehat{b}_{n}\left|u_{n}\right|^{p-2} u_{n}-\left[\int_{\Omega}\left(\left|\nabla u_{n}\right|^{\gamma}+\int_{\partial \Omega}\left|u_{n}\right|^{\gamma} d \sigma\right)^{(p / \gamma)-1} d x\right]\left|u_{n}\right|^{\gamma-2} u_{n} \\
& \quad-\widehat{d}_{n}\left|u_{n}\right|^{q_{1}-2} u_{n} \quad \text { on } \partial \Omega .
\end{aligned}
$$


Pointing out that $V^{+}\left(D_{1}\right) \neq \emptyset$, we advance the conditions

$$
\begin{gathered}
\int_{\Omega}\left(\sum_{\ell=1}^{n-1} \rho_{\ell}\right) d x<\int_{\partial \Omega} \widehat{d} d \sigma \quad\left(\widehat{d}=\min \left\{\widehat{d}_{1}, \ldots, \widehat{d}_{n}\right\}\right), \\
\int_{\Omega} b_{\ell} d x>0, \quad \int_{\partial \Omega} \widehat{b}_{\ell} d \sigma>0 \quad \text { as } \ell=1, \ldots, n-1, \\
b_{n} \geq 0, \quad \widehat{b}_{n} \geq 0 .
\end{gathered}
$$

Taking into account that

$$
(3.41) \Longrightarrow D_{1}\left(c_{1}, \ldots, c_{n-1}, 0\right)<0 \quad \forall\left(c_{1}, \ldots, c_{n-1}\right) \in R^{n-1} \backslash\{0\},
$$

we have (Propositions 3.2 and 3.4)

(3.41) and (3.43)

$\Longrightarrow\left(\right.$ with $\lambda_{n}, \mu_{n} \leq 0, \lambda_{n}+\mu_{n}<0 \exists \delta_{1}^{*}>0:\left(i_{14}\right)$ holds if $\left|\lambda_{\ell}\right|,\left|\mu_{\ell}\right| \leq \delta_{1}^{*}$ as $\left.\ell=1, \ldots, n-1\right)$,

$\Longrightarrow$ (with $\lambda_{n}, \mu_{n} \leq 0, \lambda_{n}+\mu_{n}<0 \exists \delta_{2}^{*}>0:\left(i_{15}\right)$ holds if $\lambda_{\ell}, \mu_{\ell} \in\left[0, \delta_{2}^{*}\right]$ as $\ell=1, \ldots, n-1$ and $\lambda_{\ell}+\mu_{\ell}>0$ for some $\ell$ ).

Proposition 3.13 (see ([1], Theorems 2.1 and 4.1; Remark 2.1); Proposition A.4; [5, 6]). Under assumption (3.39), we have

(i) When (3.41) and (3.43) hold, with $\lambda_{\ell}, \mu_{\ell}$ as in (3.45) system (3.40) has at least one weak solution $u^{0}\left(u^{0}=\tau^{0} v^{0}, \tau^{0}=\right.$ const. $\left.>0, v^{0} \in S_{\lambda \mu} \cap V^{+}\left(D_{1}\right)\right)$, and it results in

$$
\begin{aligned}
u_{i}^{0} \in L^{\infty}(\Omega) \cap C_{\ell o c}^{1, \alpha_{i}^{0}}(\Omega), & u_{i}^{0}>0 \text { as } i=1, \ldots, n-1, \\
u_{n}^{0} \in L^{\infty}(\Omega), & u_{n}^{0} \geq 0, \quad u_{n}^{0} \not \equiv 0
\end{aligned}
$$

(ii) When (3.41)-(3.43) hold, with $\lambda_{\ell}, \mu_{\ell}$ as in (3.46) system (3.40) has at least one weak solution $\bar{u}\left(\bar{u}=\bar{\tau} \bar{v}, \bar{\tau}=\right.$ const. $\left.>0, \bar{v} \in V_{\lambda \mu}^{-} \cap S\left(D_{1}\right)\right)$, and it results in $\bar{u}_{i} \neq 0$ as $i=1, \ldots, n$.

Consequently, when (3.41)-(3.43) hold, with $\lambda_{\ell}, \mu_{\ell}$ as in (3.46) and $\min \left\{\delta_{1}^{*}, \delta_{2}^{*}\right\}$ instead of $\delta_{2}^{*}$ system (3.40) has at least two different weak solutions.

About the properties of $u_{i}^{0}$ and $\bar{u}_{i}$ expressed by Proposition 3.13, it is necessary to remark that if $u=\left(u_{1}, \ldots, u_{n}\right)$ is a nontrivial weak solution to system (3.40), then $u_{i} \not \equiv 0$ as $i=1, \ldots, n$. In fact,

$$
u_{n} \equiv 0 \Longrightarrow u_{i} \equiv 0 \quad \text { as } i=1, \ldots, n-1, \quad u_{i} \equiv 0 \quad \text { for some } i \in\{1, \ldots, n-1\} \Longrightarrow u_{n} \equiv 0 \text {. }
$$


Application 3.14. Let $n=2$ and for any $v=\left(v_{1}, v_{2}\right) \in W$ :

$$
\begin{gathered}
A(v)=p^{-1} \sum_{\ell=1}^{2} \int_{\Omega}\left|\nabla v_{\ell}\right|^{p} d x, \quad D_{j}(v)=\left.\left.q_{j}^{-1} \int_{\Omega} \rho_{j}\left|\sum_{\ell=1}^{2} d_{j \ell}\right| v_{\ell}\right|^{\gamma_{j}}\right|^{q_{j} / \gamma_{j}} d x \quad \text { as } j=1, \ldots, m-1, \\
D_{m}(v)=q_{m}^{-1} \int_{\partial \Omega} \rho_{m}\left(\sum_{\ell=1}^{2}\left|v_{\ell}\right|^{\gamma_{m}}\right)^{q_{m} / \gamma_{m}} d \sigma,
\end{gathered}
$$

where

$$
\begin{gathered}
1<\gamma_{j}<q_{j} \quad \text { as } j=1, \ldots, m, \quad p<q_{1}<\cdots<q_{m}<\hat{p}, \quad \rho_{j} \in L^{\infty}(\Omega), \quad \rho_{j}<0, \\
d_{j \ell} \in L^{\infty}(\Omega) \backslash\{0\}, \quad \rho_{m} \in L^{\infty}(\partial \Omega) .
\end{gathered}
$$

Let us consider the system:

$$
\begin{aligned}
-\operatorname{div}\left(\left|\nabla u_{i}\right|^{p-2} \nabla u_{i}\right)= & \lambda_{i} b_{i}\left|u_{i}\right|^{p-2} u_{i} \\
& +\left.\left.\sum_{j=1}^{m-1} \rho_{j}\left|\sum_{\ell=1}^{2} d_{j \ell}\right| u_{\ell}\right|^{\gamma_{j}}\right|^{\left(q_{j} / \gamma_{j}\right)-2}\left(\sum_{\ell=1}^{2} d_{j \ell}\left|u_{\ell}\right|^{\gamma_{j}}\right) d_{j i}\left|u_{i}\right|^{\gamma_{j}-2} u_{i} \quad \text { in } \Omega, \\
\left|\nabla u_{i}\right|^{p-2} \frac{\partial u_{i}}{\partial v}= & \mu_{i} \widehat{b}_{i}\left|u_{i}\right|^{p-2} u_{i}+\rho_{m}\left(\sum_{\ell=1}^{2}\left|u_{\ell}\right|^{\gamma_{m}}\right)^{\left(q_{m} / \gamma_{m}\right)-1}\left|u_{i}\right|^{\gamma_{m}-2} u_{i} \quad \text { on } \partial \Omega \text { as } i=1,2 .
\end{aligned}
$$

Let us introduce the conditions:

$$
\begin{gathered}
\rho_{m}^{+} \not \equiv 0 \quad\left(\Longrightarrow V^{+}\left(D_{m}\right) \neq \emptyset \text { (Proposition A.2) }\right), \\
\int_{\partial \Omega} \rho_{m} d \sigma<0 \quad\left(\Longrightarrow D_{m}\left(c_{1}, c_{2}\right)<0 \forall\left(c_{1}, c_{2}\right) \in R^{2} \backslash\{0\}\right), \\
\int_{\Omega} b_{\ell} d x>0, \quad \int_{\partial \Omega} \widehat{b}_{\ell} d \sigma>0 \quad \text { as } \ell=1,2,
\end{gathered}
$$

we have (Propositions 3.3 and 3.5)

(3.52) and (3.53) $\Longrightarrow\left(\exists \delta_{1}^{*}>0:\left(i_{14}\right)\right.$ holds if $\left|\lambda_{\ell}\right|,\left|\mu_{\ell}\right| \leq \delta_{1}^{*}$ as $\left.\ell=1,2\right)$,

(3.53) and (3.54)

$\Longrightarrow\left(\exists \delta_{2}^{*}>0:\left(i_{15}\right)\right.$ holds if $\lambda_{\ell}, \mu_{\ell} \in\left[0, \delta_{2}^{*}\right] \quad$ as $\ell=1,2, \lambda_{\ell}+\mu_{\ell}>0$ for some $\left.\ell\right)$.

Proposition 3.15 (see ([1], Theorems 2.2 and 4.2; Remarks 2.3 and 4.4); Proposition A.4; [5]). Under assumption (3.50), we have 
(i) When (3.52) and (3.53) hold, with $\lambda_{\ell}, \mu_{\ell}$ as in (3.55) system (3.51) has at least two weak solutions $u^{0}$ and $-u^{0}\left(u^{0}=\tau^{0} v^{0}, \tau^{0}=\right.$ const. $\left.>0, v^{0} \in S_{\lambda \mu} \cap V^{+}\left(D_{m}\right)\right)$, and it results in

$$
u_{i}^{0} \in L^{\infty}(\Omega) \cap C_{\ell \circ C}^{1, \alpha_{i}^{0}}(\Omega), \quad u_{i}^{0} \geq 0 \quad \text { as } i=1,2 ;
$$

(ii) When (3.53) and (3.54) hold, with $\lambda_{\ell}, \mu_{\ell}$ as in (3.56) system (3.51) has at least two weak solutions $\bar{u}$ and $-\bar{u}\left(\bar{u}=\bar{\tau} \bar{v}, \bar{\tau}=\right.$ const. $\left.>0, \bar{v} \in V_{\lambda \mu}^{-} \cap S\left(D_{m}\right)\right)$, and it results in

$$
\bar{u}_{i} \in L^{\infty}(\Omega) \cap C_{\ell \circ c}^{1, \bar{x}_{i}}(\Omega), \quad \bar{u}_{i} \geq 0 \quad \text { as } i=1,2 .
$$

Consequently, when (3.52)-(3.54) hold, with $\lambda_{\ell}, \mu_{\ell}$ as in (3.56), and $\min \left\{\delta_{1}^{*}, \delta_{2}^{*}\right\}$ instead of $\delta_{2}^{*}$ system (3.51) has at least four different weak solutions.

Proposition 3.16. Under the assumption $p \leq 2 \gamma_{j}$ and $d_{j 1} \cdot d_{j 2}<0$ as $j=1, \ldots, m-1$, we have

(i) if $\gamma_{j_{0}}<p$ for some $j_{0} \in\{1, \ldots, m\}$, then $u_{i}^{0}>0$ as $i=1,2$;

(ii) if $\gamma_{j_{0}}<\gamma_{m} \leq p$ for some $j_{0} \in\{1, \ldots, m-1\}$, then $\bar{u}_{i}>0$ as $i=1,2$.

Proof. First of all $u_{i}^{0}$ is a weak supersolution to the equation:

$$
-\operatorname{div}\left(\left|\nabla u_{i}\right|^{p-2} \nabla u_{i}\right)=\lambda_{i} b_{i}\left|u_{i}\right|^{p-2} u_{i}+\sum_{j=1}^{m-1} \rho_{j}\left|d_{j 1}\left(u_{1}^{0}\right)^{\gamma_{j}}+d_{j 2}\left(u_{2}^{0}\right)^{\gamma_{j}}\right|^{\left(q_{j} / \gamma_{j}\right)-2} d_{j i}^{2}\left|u_{i}\right|^{2 \gamma_{j}-2} u_{i} \quad \text { in } \Omega .
$$

Also, $\bar{u}_{i}$ has a similar property. Then [6] it is sufficient to verify that

$$
\begin{aligned}
& u_{i}^{0} \neq 0, \\
& \bar{u}_{i} \not \equiv 0 .
\end{aligned}
$$

About (3.60), let us prove (Remark 1.1) that

$$
\left(i_{16}^{h}\right) \text { holds as } h=1,2 \text { with } \mathfrak{F}=S_{\lambda \mu} \cap V^{+}\left(D_{m}\right)
$$

Let $v=\left(v_{1}, v_{2}\right) \in V^{+}\left(D_{m}\right) \cap S_{\lambda \mu}$. Let, for example, $v_{1} \equiv 0$. Let

$$
\begin{gathered}
\mathbb{K} \subseteq \Omega \text { a compact set : }|\mathbb{K}|_{N}>0, \quad v_{2} \not \equiv 0 \text { in } \mathbb{K}, \\
\Omega^{\prime} \text { an open set : } \mathbb{K} \subseteq \Omega^{\prime}, \quad \overline{\Omega^{\prime}} \subseteq \Omega, \\
\Gamma \subseteq \partial \Omega: \sigma(\Gamma)>0, \quad \rho_{m}\left|v_{2}\right|>0 \quad \text { on } \Gamma .
\end{gathered}
$$


Since Propositions A.1 and A.2, there exists $\varphi \in C_{0}^{\infty}\left(R^{N}\right)$, with $0 \leq \varphi \leq 1$ and supp $\varphi \subseteq$ $\Omega^{\prime} \cup\left(R^{N} \backslash \overline{\Omega^{\prime}}\right)$, such that

$$
\begin{gathered}
\left.\left.\int_{\Omega} \rho_{j}\left|d_{j 2}\right| v_{2}\right|^{\gamma_{j}}\right|^{\left(q_{j} / \gamma_{j}\right)-2}\left|v_{2}\right|^{\gamma_{j}} \varphi^{\gamma_{j}} d_{j 1} d_{j 2} d x>0 \quad \text { as } j=1, \ldots, m-1, \quad \int_{\partial \Omega} \rho_{m}\left|v_{2}\right|^{q_{m}-\gamma_{m}} \varphi^{\gamma_{m}} d \sigma>0, \\
\delta=p^{-1}\left[\int_{\Omega}|\nabla \varphi|^{p} d x-\lambda_{1} \int_{\Omega} b_{1} \varphi^{p} d x-\mu_{1} \int_{\partial \Omega} \widehat{b}_{1} \varphi^{p} d \sigma\right]>0 .
\end{gathered}
$$

Then with $v(s)=\left((1-s)^{1 / p} \delta^{-1 / p} \varphi, s^{1 / p} v_{2}\right)$, we have

$$
\begin{gathered}
H_{\lambda \mu}(v(s))=1 \quad \forall s \in[0,1], \quad \exists s_{0} \in\left[0,1\left[: D_{m}(v(s))>0 \quad \forall s \in\left[s_{0}, 1\right],\right.\right. \\
\lim _{s \rightarrow 1^{-}} \frac{d}{d s} D_{j_{0}}(v(s))=-\infty, \quad \lim _{s \rightarrow 1^{-}} \frac{d}{d s} D_{j}(v(s))<+\infty \quad \text { as } j \neq j_{0} .
\end{gathered}
$$

Passing to (3.61), let us introduce the function $\psi(t, v)=p t^{p-1} H_{\lambda \mu}(v)-\sum_{j=1}^{m} q_{j} t^{q_{j}-1} D_{j}(v)$, and let us remember that ([1], Theorem 4.2)

$$
\begin{gathered}
\forall v \in V_{\lambda \mu}^{-} \cap S\left(D_{m}\right) \exists \mid t(v)>0: \psi(t(v), v)=0, \\
\widetilde{\widetilde{E}}(\bar{v})=\inf \left\{\widetilde{\widetilde{E}}(v): v \in V_{\lambda \mu}^{-} \cap S\left(D_{m}\right)\right\},
\end{gathered}
$$

where $\tilde{\widetilde{E}}(v)=(t(v))^{p} H_{\lambda \mu}(v)-\sum_{j=1}^{m}(t(v))^{q_{j}} D_{j}(v)$.

Reasoning by contradiction, let us set, for example, $\bar{v}_{1} \equiv 0$ and set $v(s)=$ $\left((1-s)^{1 / \gamma_{m}} \bar{v}_{2}, s^{1 / \gamma_{m}} \bar{v}_{2}\right)$. Since

$$
\begin{gathered}
D_{m}(v(s))=-1 \quad \forall s \in[0,1], \quad \exists s_{0} \in\left[0,1\left[: H_{\lambda \mu}(v(s))<0 \quad \forall s \in\left[s_{0}, 1\right],\right.\right. \\
\lim _{s \rightarrow 1^{-}} \frac{d}{d s} D_{j_{0}}(v(s))=-\infty, \quad \lim _{s \rightarrow 1^{-}} \frac{d}{d s} D_{j}(v(s))<+\infty \quad \text { as } j \in\{1, \ldots, m-1\} \backslash\left\{j_{0}\right\},
\end{gathered}
$$

as in Proposition 2.24, we get the contradiction:

$$
\widetilde{\widetilde{E}}(\overline{\mathcal{v}}) \leq \widetilde{\widetilde{E}}(v(s))<\widetilde{\widetilde{E}}(\bar{v}) \quad \forall s \in\left[s_{1}, 1\left[\left(s_{0} \leq s_{1}<1\right) .\right.\right.
$$

Application 3.17. Let $n>2$ and set for each $v=\left(v_{1}, \ldots, v_{n}\right) \in W$ :

$$
\begin{gathered}
A(v)=p^{-1} \sum_{\ell=1}^{n} \int_{\Omega}\left|\nabla v_{\ell}\right|^{p} d x, \\
D_{j}(v)=\left.q_{j}^{-1} \sum_{\substack{\ell=1 \\
\ell \neq j}}^{n} \int_{\Omega} \rho_{j}\left|d_{j j}\right| v_{j}\right|^{\gamma_{j}}+\left.d_{j \ell}\left|v_{\ell}\right|^{\gamma_{j}}\right|^{q_{j} / \gamma_{j}} d x \quad \text { as } j=1, \ldots, n, \\
D_{n+1}(v)=q_{n+1}^{-1} \int_{\partial \Omega} \rho_{n+1}\left(\sum_{\ell=1}^{n}\left|v_{\ell}\right|^{\gamma_{n+1}}\right)^{q_{n+1} / \gamma_{n+1}} d \sigma,
\end{gathered}
$$


where

$$
\begin{gathered}
1<\gamma_{j}<q_{j} \text { as } j=1, \ldots, n+1, \quad p<q_{1}<\cdots<q_{n+1}<\hat{p}, \quad \rho_{j} \in L^{\infty}(\Omega), \quad \rho_{j}<0, \\
d_{j l} \in L^{\infty}(\Omega) \backslash\{0\}, \quad \rho_{n+1} \in L^{\infty}(\partial \Omega) .
\end{gathered}
$$

Let us consider the system:

$$
\begin{aligned}
& -\operatorname{div}\left(\left|\nabla u_{i}\right|^{p-2} \nabla u_{i}\right)=\lambda_{i} b_{i}\left|u_{i}\right|^{p-2} u_{i}+\left.\sum_{\ell \neq i} \rho_{i}\left|d_{i i}\right| u_{i}\right|^{\gamma_{i}}+\left.d_{i \ell}\left|u_{\ell}\right|^{\gamma_{i}}\right|^{\left(q_{i} / \gamma_{i}\right)-2}\left(d_{i i}\left|u_{i}\right|^{\gamma_{i}}+d_{i \ell}\left|u_{\ell}\right|^{\gamma_{i}}\right) d_{i i}\left|u_{i}\right|^{\gamma_{i}-2} u_{i} \\
& \quad+\left.\sum_{j \neq i} \rho_{j}\left|d_{j j}\right| u_{j}\right|^{\gamma_{j}}+\left.d_{j i}\left|u_{i}\right|^{\gamma_{j}}\right|^{\left(q_{j} / \gamma_{j}\right)-2}\left(d_{j j}\left|u_{j}\right|^{\gamma_{j}}+d_{j i}\left|u_{i}\right|^{\gamma_{j}}\right) d_{j i}\left|u_{i}\right|^{\gamma_{j}-2} u_{i} \quad \text { in } \Omega, \\
& \left|\nabla u_{i}\right|^{p-2} \frac{\partial u_{i}}{\partial v}=\mu_{i} \widehat{b}_{i}\left|u_{i}\right|^{p-2} u_{i}+\rho_{n+1}\left(\sum_{\ell=1}^{n}\left|u_{\ell}\right|^{\gamma_{n+1}}\right)^{\left(q_{n+1} / \gamma_{n+1}\right)-1}\left|u_{i}\right|^{\gamma_{n+1}-2} u_{i} \quad \text { on } \partial \Omega \text { as } i=1, \ldots, n .
\end{aligned}
$$

Let us make the assumptions:

$$
\rho_{n+1}^{+} \not \equiv 0, \quad \int_{\partial \Omega} \rho_{n+1} d \sigma<0, \quad \int_{\Omega} b_{\ell} d x>0, \quad \int_{\partial \Omega} \widehat{b}_{\ell} d \sigma>0 \quad \text { as } \ell=1, \ldots, n .
$$

About Neumann's problem (3.71), we have an existence result similar to the one of Proposition 3.15 related to system (3.51). About the positive sign of the components of the weak solutions $u^{0}$ and $\bar{u}$ to system (3.71), as in Proposition 3.16, we show.

Proposition 3.18. Under the assumption $p \leq 2 \gamma_{j}$ as $j=1, \ldots, n$ and $d_{j j} \cdot d_{j \ell}<0$ as $j, \ell \in\{1, \ldots, n\}$ with $\ell \neq j$, we have

(i) if either $\gamma_{n+1}<p$ or $\gamma_{j}<p$ for all $j \in\{1, \ldots, n\} \backslash\left\{j_{0}\right\}$ for some $j_{0}$, then $u_{i}^{0}>0$ as $i=1, \ldots, n$;

(ii) if $\gamma_{j}<\gamma_{n+1} \leq p$ for all $j \in\{1, \ldots, n\} \backslash\left\{j_{0}\right\}$ for some $j_{0}$, then $\bar{u}_{i}>0$ as $i=1, \ldots, n$.

The following remark deals also with Application 3.14.

Remark 3.19. Making in (3.50) (resp. (3.70)) the change

$$
q_{1}<\cdots<q_{m}<p \quad\left[\text { resp. } q_{1}<\cdots<q_{n+1}<p\right]
$$

system (3.51) (resp. (3.71)) has at least the two weak solutions $\bar{u}$ and $-\bar{u}$ ([1], Theorem 4.2; Remark 4.4). The components of $\bar{u}$ keep the properties that Propositions 3.15 and 3.16 (Proposition 3.15 and Proposition 3.18 resp.) underline. 
Application 3.20. Let for each $v=\left(v_{1}, \ldots, v_{n}\right) \in W$ :

$$
\begin{gathered}
A(v)=p^{-1} \sum_{\ell=1}^{n} \int_{\Omega}\left|\nabla v_{\ell}\right|^{p} d x, \\
D_{j}(v)=\left.q_{j}^{-1} \int_{\partial \Omega} \rho_{j}\left|d_{j 1}\right| v_{j}\right|^{\gamma_{j}}+\left.d_{j 2}\left|v_{n}\right|^{\gamma_{j}}\right|^{q_{j} / \gamma_{j}} d \sigma \quad \text { as } j=1, \ldots, n-1, \\
D_{n}(v)=\left(\int_{\partial \Omega}\left|v_{n}\right|^{\hat{\gamma}_{n}} d \sigma\right)\left(\int_{\Omega} \rho_{n}\left|v_{n}\right|^{\gamma_{n}} d x\right),
\end{gathered}
$$

where

$$
\begin{aligned}
& 1<\gamma_{j}<q_{j}<\hat{p} \quad \text { as } j=1, \ldots, n-1, \quad 1<\gamma_{n}<\tilde{p}, \quad 1<\widehat{\gamma}_{n}<\hat{p}, \\
& p<q_{1}<\cdots<q_{n-1}<q_{n}=\gamma_{n}+\widehat{\gamma}_{n}, \\
& \rho_{j} \in L^{\infty}(\partial \Omega), \quad \rho_{j}<0, \quad d_{j 1}, d_{j 2} \in L^{\infty}(\partial \Omega) \backslash\{0\}, \quad \rho_{n} \in L^{\infty}(\Omega) \text {. }
\end{aligned}
$$

Let us consider the system:

$$
\begin{aligned}
-\operatorname{div}\left(\left|\nabla u_{i}\right|^{p-2} \nabla u_{i}\right)= & \lambda_{i} b_{i}\left|u_{i}\right|^{p-2} \text { in } \Omega \text { as } i=1, \ldots, n-1, \\
-\operatorname{div}\left(\left|\nabla u_{n}\right|^{p-2} \nabla u_{n}\right)= & \lambda_{n} b_{n}\left|u_{n}\right|^{p-2} u_{n}+\gamma_{n}\left(\int_{\partial \Omega}\left|u_{n}\right|^{\widehat{\gamma}_{n}} d \sigma\right) \rho_{n}\left|u_{n}\right|^{\gamma_{n}-2} u_{n} \quad \text { in } \Omega, \\
\left|\nabla u_{i}\right|^{p-2} \frac{\partial u_{i}}{\partial v}= & \mu_{i} \widehat{b}_{i}\left|u_{i}\right|^{p-2} u_{i}+\left.\rho_{i}\left|d_{i 1}\right| u_{i}\right|^{\gamma_{i}}+\left.d_{i 2}\left|u_{n}\right|^{\gamma_{i}}\right|^{\left(q_{i} / \gamma_{i}\right)-2} \\
& \times\left(d_{i 1}\left|u_{i}\right|^{\gamma_{i}}+d_{i 2}\left|u_{n}\right|^{\gamma_{i}}\right) d_{i 1}\left|u_{i}\right|^{\gamma_{i}-2} u_{i} \quad \text { on } \partial \Omega \text { as } i=1, \ldots, n-1, \\
\left|\nabla u_{n}\right|^{p-2} \frac{\partial u_{n}}{\partial v}= & \mu_{n} \widehat{b}_{n}\left|u_{n}\right|^{p-2} u_{n}+\left.\sum_{j=1}^{n-1} \rho_{j}\left|d_{j 1}\right| u_{j}\right|^{\gamma_{j}}+\left.d_{j 2}\left|u_{n}\right|^{\gamma_{j}}\right|^{\left(q_{j} / \gamma_{j}\right)-2} \\
& \times\left(d_{j 1}\left|u_{j}\right|^{\gamma_{j}}+d_{j 2}\left|u_{n}\right|^{\gamma_{j}}\right) d_{j 2}\left|u_{n}\right|^{\gamma_{j}-2} u_{n} \\
& +\widehat{\gamma}_{n}\left(\int_{\Omega} \rho_{n}\left|u_{n}\right|^{\gamma_{n}} d x\right)\left|u_{n}\right|^{\gamma_{n}-2} u_{n} \quad \text { on } \partial \Omega .
\end{aligned}
$$

Let us introduce the conditions:

$$
\begin{gathered}
\rho_{n}^{+} \not \equiv 0 \quad\left(\Longrightarrow V^{+}\left(D_{n}\right) \neq \emptyset(\text { Propositions A.1 and A.2 })\right) \\
\int_{\Omega} \rho_{n} d x<0 \quad\left(\Longrightarrow D_{n}\left(0, \ldots, 0, c_{n}\right)<0 \forall c_{n} \in R^{n} \backslash\{0\}\right), \\
b_{\ell} \geq 0, \quad \widehat{b}_{\ell} \geq 0 \quad \text { as } \ell=1, \ldots, n-1, \\
\int_{\Omega} b_{n} d x>0, \quad \int_{\partial \Omega} \widehat{b}_{n} d \sigma>0 .
\end{gathered}
$$


We have (Propositions 3.2 and 3.4)

$$
\begin{aligned}
& \text { (3.77)-(3.79) } \\
& \Longrightarrow\left(\text { with } \lambda_{\ell}, \mu_{\ell} \leq 0, \lambda_{\ell}+\mu_{\ell}<0 \text { as } \ell=1, \ldots, n-1 \exists \delta_{1}^{*}>0:\right. \\
& \left.\quad\left(i_{14}\right) \text { holds if }\left|\lambda_{n}\right|,\left|\mu_{n}\right| \leq \delta_{1}^{*}\right) \\
& (3.78)-(3.80) \\
& \Longrightarrow\left(\text { with } \lambda_{\ell}, \mu_{\ell} \leq 0, \lambda_{\ell}+\mu_{\ell}<0 \text { as } \ell=1, \ldots, n-1\right. \\
& \left.\quad \exists \delta_{2}^{*}>0:\left(i_{15}\right) \text { holds if } \lambda_{n}, \mu_{n} \in\left[0, \delta_{2}^{*}\right] \text { and } \lambda_{n}+\mu_{n}>0\right)
\end{aligned}
$$

Proposition 3.21 (see ([1], Theorems 2.2 and 4.2; Remarks 2.3 and 4.4); Proposition A.4; [5, 6]). Under assumption (3.75), we have

(i) When (3.77)-(3.79) hold, with $\lambda_{\ell}, \mu_{\ell}$ as in (3.81), system (3.76) has at least two weak solutions $u^{0}$ and $-u^{0}\left(u^{0}=\tau^{0} v^{0}, \tau^{0}=\right.$ const. $\left.>0, v^{0} \in S_{\lambda \mu} \cap V^{+}\left(D_{n}\right)\right)$, and it results in $u_{i}^{0} \geq 0(i=1, \ldots, n), u_{n}^{0} \not \equiv 0$. If $\gamma_{n}<\hat{p}$, then

$$
\begin{gathered}
u_{i}^{0} \in L^{\infty}(\Omega) \cap C_{\ell \circ c}^{1, \alpha_{i}^{0}}(\Omega) \quad(i=1, \ldots, n), \\
u_{i}^{0} \not \equiv 0 \Longrightarrow u_{i}^{0}>0 \quad(i=1, \ldots, n-1) ;
\end{gathered}
$$

(ii) When (3.78)-(3.80) hold, with $\lambda_{\ell}, \mu_{\ell}$ as in (3.82), system (3.76) has at least two weak solutions $\bar{u}$ and $-\bar{u}\left(\bar{u}=\overline{\tau v}, \bar{\tau}=\right.$ const. $\left.>0, \bar{v} \in V_{\bar{\lambda}}^{-} \cap S\left(D_{n}\right)\right)$, and it results in $\bar{u}_{i} \geq 0(i=1, \ldots, n), \bar{u}_{n} \neq 0$. If $\gamma_{n}<\hat{p}$, then

$$
\begin{gathered}
\bar{u}_{i} \in L^{\infty}(\Omega) \cap C_{\ell o c}^{1, \bar{\alpha}_{i}}(\Omega) \quad(i=1, \ldots, n), \\
\bar{u}_{i} \equiv 0 \Rightarrow \bar{u}_{i}>0 \quad(i=1, \ldots, n-1) .
\end{gathered}
$$

Consequently, when (3.77)-(3.80) hold, with $\lambda_{\ell}, \mu_{\ell}$ as in (3.82), and $\min \left\{\delta_{1}^{*}, \delta_{2}^{*}\right\}$ instead of $\delta_{2}^{*}$ system (3.76) has at least four different weak solutions. Obviously, $u_{n}^{0}>0$ and $\bar{u}_{n}>0$ if $p \leq \gamma_{n}<\hat{p}$.

The following proposition gives a sufficient condition to

$$
\begin{aligned}
& u_{i}^{0}>0 \quad \text { as } i=1, \ldots, n-1, \\
& \bar{u}_{i}>0 \quad \text { as } i=1, \ldots, n-1 .
\end{aligned}
$$

Proposition 3.22. Let $\gamma_{n}<\hat{p}$. If $\gamma_{j}<p$ and $d_{j 1} \cdot d_{j 2}<0$ as $j=1, \ldots, n-1$, then (3.85) and (3.86) hold.

Proof. Since

$$
\left(v_{1}, \ldots, v_{n}\right) \in V^{+}\left(D_{n}\right) \Longrightarrow\left(\exists \Gamma \subseteq \partial \Omega: \sigma(\Gamma)>0,\left|v_{n}\right|>0 \text { on } \Gamma\right)
$$


using Propositions A.1 and A.2, we can verify that

$$
\left(i_{16}^{h}\right) \text { holds as } h=1, \ldots, n-1 \text { with } \mathfrak{F}=S_{\lambda \mu} \cap V^{+}\left(D_{n}\right) \text {, }
$$

from which (Remark 1.1) we get (3.85).

Let us prove (3.86). Reasoning by contradiction, let us set, for example, $\bar{v}_{1} \equiv 0$. If $v(s)=\left((1-s)^{1 / p} \bar{v}_{n}, \bar{v}_{2}, \ldots, \bar{v}_{n}\right)$, we have

$$
\begin{gathered}
D_{n}(v(s))=-1 \quad \forall s \in[0,1], \quad \exists s_{0} \in\left[0,1\left[: H_{\lambda \mu}(v(s))<0 \quad \forall s \in\left[s_{0}, 1\right],\right.\right. \\
\lim _{s \rightarrow 1^{-}} \frac{d}{d s} D_{1}(v(s))=-\infty .
\end{gathered}
$$

Then as in Proposition 3.16, we get a contradiction.

Remark 3.23. Making in (3.75) the change:

$$
1<\gamma_{j}<q_{j} \quad \text { as } j=1, \ldots, n-1, \quad 1<\gamma_{n}, \quad 1<\widehat{\gamma}_{n}, \quad q_{1}<\cdots<q_{n}=\gamma_{n}+\widehat{\gamma}_{n}<p,
$$

system (3.76) has at least the two weak solutions $\bar{u}$ and $-\bar{u}$ ([1], Theorem 4.2; Remark 4.4). The components of $\bar{u}$, all bounded, are locally Hölderian with their first derivatives. If $d_{j 1}$. $d_{j 2}<0$ as $j=1, \ldots, n-1$, then (3.86) holds.

Application 3.24. Let for each $v=\left(v_{1}, \ldots, v_{n}\right) \in W$ :

$$
\begin{gathered}
A(v)=p^{-1} \sum_{\ell=1}^{n} \int_{\Omega}\left(\left|\nabla v_{\ell}\right|^{\gamma}+\int_{\Omega}\left|v_{\ell}\right|^{\gamma} d x\right)^{p / \gamma} d x, \\
D_{j}(v)=q_{j}^{-1} \int_{\partial \Omega}\left(\sum_{\ell=1}^{n}\left|v_{\ell}\right|^{\gamma}\right)^{q_{j} / \gamma} d \sigma \quad \text { as } j=1, \ldots, m-1, \quad D_{m}(v)=q_{m}^{-1} \int_{\Omega}\left(\sum_{\ell=1}^{n}\left|v_{\ell}\right|^{\gamma}\right)^{q_{m} / \gamma} d x,
\end{gathered}
$$

where

$$
1<\gamma<q_{1}<\cdots<q_{m}<p
$$


Let us consider the system:

$$
\begin{aligned}
-\operatorname{div} & {\left[\left(\left|\nabla u_{i}\right|^{\gamma}+\int_{\Omega}\left|u_{i}\right|^{\gamma} d x\right)^{(p / \gamma)-1}\left|\nabla u_{i}\right|^{\gamma-2} \nabla u_{i}\right] } \\
= & \lambda_{i} b_{i}\left|u_{i}\right|^{p-2} u_{i}-\left(\int_{\Omega}\left(\left|\nabla u_{i}\right|^{\gamma}+\int_{\Omega}\left|u_{i}\right|^{\gamma} d x\right)^{(p / \gamma)-1} d x\right)\left|u_{i}\right|^{\gamma-2} u_{i} \\
& +\left(\sum_{\ell=1}^{n}\left|u_{\ell}\right|^{\gamma}\right)^{\left(q_{m} / \gamma\right)-1}\left|u_{i}\right|^{\gamma-2} u_{i} \text { in } \Omega, \\
\left(\left|\nabla u_{i}\right|^{\gamma}+\int_{\Omega}\left|u_{i}\right|^{\gamma} d x\right)^{(p / \gamma)-1}\left|\nabla u_{i}\right|^{\gamma-2} \frac{\partial u_{i}}{\partial v} & \\
= & \mu_{i} \widehat{b}_{i}\left|u_{i}\right|^{p-2} u_{i}+\sum_{j=1}^{m-1}\left(\sum_{\ell=1}^{n}\left|u_{\ell}\right|^{\gamma}\right)^{\left(q_{j} / \gamma\right)-1}\left|u_{i}\right|^{\gamma-2} u_{i} \quad \text { on } \partial \Omega \text { as } i=1, \ldots n .
\end{aligned}
$$

We advance the condition:

$$
b_{\ell} \geq 0, \quad \widehat{b}_{\ell} \geq 0 \quad \text { as } \ell=1, \ldots n,
$$

and we note that (Proposition 3.1)

$$
\text { (3.94) } \Longrightarrow\left(\left(i_{13}\right) \text { holds if } \lambda_{\ell}, \mu_{\ell} \leq 0, \lambda_{\ell}+\mu_{\ell}<0 \quad \text { as } \ell=1, \ldots, n\right) \text {. }
$$

Proposition 3.25. Under conditions (3.92) and (3.94), with $\lambda_{\ell}, \mu_{\ell}$ as in (3.95), system (3.93) has at least two weak solutions $u^{0}$ and $-u^{0}\left(u^{0}=\tau^{0} v^{0}, \tau^{0}=\right.$ const. $\left.>0, v^{0} \in S^{+}\left(D_{1}, \ldots, D_{m}\right)\right)$, and it results in

$$
u_{i}^{0} \in L^{\infty}(\Omega), u_{i}^{0} \geq 0, \quad u_{i}^{0} \not \equiv 0 \quad \text { as } i=1, \ldots, n .
$$

Proof. We recall that ([1], Section 2), set $\psi(t, v)=p t^{p-1} H_{\lambda \mu}(v)-\sum_{j=1}^{m} q_{j} t^{q_{j}-1} D_{j}(v)$, we have

$$
\begin{gathered}
\forall v \in V^{+}\left(D_{1}, \ldots, D_{m}\right) \quad \exists \mid t(v)>0: \psi(t(v), v)=0, \\
\text { the functional } t(v) \text { is } C^{1} \text { in } V^{+}\left(D_{1}, \ldots, D_{m}\right) .
\end{gathered}
$$

We introduce the functional $\widetilde{\widetilde{E}}(v)=(t(v))^{p} H_{\lambda \mu}(v)-\sum_{j=1}^{m}(t(v))^{q_{j}} D_{j}(v)$ which is $C^{1}$ in $V^{+}\left(D_{1}, \ldots, D_{m}\right)$. We still remember that ([1], Theorem 2.3; Remark 2.5)

$$
\begin{gathered}
\exists v^{0} \in S^{+}\left(D_{1}, \ldots, D_{m}\right), \quad \text { with } v_{i}^{0} \geq 0 \text { as } i=1, \ldots, n, \text { such that } \\
\widetilde{\widetilde{E}}\left(v^{0}\right)=\inf \left\{\widetilde{\widetilde{E}}(v): v \in S^{+}\left(D_{1}, \ldots, D_{m}\right)\right\} \\
u^{0}=t\left(v^{0}\right) v^{0} \text { is a weak solution to system (3.93). }
\end{gathered}
$$


The property $u_{i}^{0} \in L^{\infty}(\Omega)$ is due to Proposition A.4. Let us verify that $u_{i}^{0} \not \equiv 0$ as $i=$ $1, \ldots, n$. Reasoning by contradiction, let us set, for example, $v_{1}^{0} \equiv 0$ and $v_{2}^{0} \neq 0$. As $v(s)=$ $\left((1-s)^{1 / \gamma} v_{2}^{0}, s^{1 / \gamma} v_{2}^{0}, v_{3}^{0}, \ldots, v_{n}^{0}\right)$, we have

$$
\sum_{j=1}^{m} D_{j}(v(s))=1 \quad \forall s \in[0,1], \quad\left[\frac{d}{d s} H_{\lambda \mu}(v(s))\right]_{s=1}>0
$$

Then, since $(d / d s) \tilde{\widetilde{E}}(v(s))=(t(v(s)))^{p}(d / d s) H_{\lambda \mu}(v(s))$, there exists $s_{0} \in[0,1[$ such that $(d / d s) \tilde{\widetilde{E}}(v(s))>0$ for all $s \in\left[s_{0}, 1\right]$, from which the contradiction:

$$
\widetilde{\widetilde{E}}\left(v^{0}\right) \leq \widetilde{\widetilde{E}}(v(s))<\widetilde{\widetilde{E}}\left(v^{0}\right), \quad \forall s \in\left[s_{0}, 1[.\right.
$$

Application 3.26. Let for each $v=\left(v_{1}, \ldots, v_{n}\right) \in W$ :

$$
\begin{gathered}
A(v)=p^{-1} \sum_{\ell=1}^{n} \int_{\Omega}\left|\nabla v_{\ell}\right|^{p} d x, \quad D_{1}(v)=q_{1}^{-1} \int_{\Omega} \rho\left|\sum_{\ell=1}^{n} d_{\ell} v_{\ell}\right|^{q_{1}} d x, \\
D_{2}(v)=\left(\int_{\Omega}\left[\sum_{\ell=1}^{n} \tilde{d}_{\ell}\left|v_{\ell}\right|^{\gamma}\right] d x\right)\left(\int_{\partial \Omega} \hat{\rho}\left[\sum_{\ell=1}^{n} \widehat{d}_{\ell}\left|v_{\ell}\right|^{\widehat{\gamma}}\right] d \sigma\right),
\end{gathered}
$$

where

$$
\begin{array}{cl}
1<\gamma<\tilde{p}, \quad 1<\hat{\gamma}<\hat{p}, \quad 1<q_{1}<\min \left\{\tilde{p}, q_{2}=\gamma+\hat{\gamma}\right\}, \\
p<q_{2}, \quad \rho, d_{\ell} \in L^{\infty}(\Omega) \backslash\{0\}, \quad \rho \leq 0, \rho d_{\ell} \not \equiv 0
\end{array}
$$

as some $\ell, \tilde{d}_{\ell} \in L^{\infty}(\Omega) \backslash\{0\}, \quad \tilde{d}_{\ell} \geq 0, \quad \hat{\rho} \in L^{\infty}(\Omega) \backslash\{0\}, \quad \hat{d}_{\ell}=$ const. $>0$.

Let as $\ell=1, \ldots, n F_{\ell}=f_{\ell}+\widehat{f}_{\ell}$, where $f_{\ell} \in L^{p^{\prime}}(\Omega)\left(p^{\prime}=p /(p-1)\right)$ and $\widehat{f}_{\ell} \in\left(W^{1-(1 / p), p}(\partial \Omega)\right)^{*}$ (dual space of $W^{1-(1 / p), p}(\partial \Omega)$ ). Let $\langle\langle F, v\rangle\rangle=\sum_{\ell=1}^{n}\left\langle F_{\ell}, v_{\ell}\right\rangle$ for all $v=\left(v_{1}, \ldots, v_{n}\right) \in W$. Let us consider the system:

$$
\begin{aligned}
& -\operatorname{div}\left(\left|\nabla u_{i}\right|^{p-2} \nabla u_{i}\right)=\lambda_{i} b_{i}\left|u_{i}\right|^{p-2} u_{i}+\rho\left|\sum_{\ell=1}^{n} d_{\ell} u_{\ell}\right|^{q_{1}-2}\left(\sum_{\ell=1}^{n} d_{\ell} u_{\ell}\right) d_{i} \\
& \quad+\gamma\left(\int_{\partial \Omega} \hat{\rho}\left[\sum_{\ell=1}^{n} \widehat{d}_{\ell}\left|u_{\ell}\right|^{\widehat{\gamma}}\right] d \sigma\right) \tilde{d}_{i}\left|u_{i}\right|^{\gamma-2} u_{i}+f_{i} \text { in } \Omega, \\
& \left|\nabla u_{i}\right|^{p-2} \frac{\partial u_{i}}{\partial v} \\
& =\mu_{i} \widehat{b}_{i}\left|u_{i}\right|^{p-2} u_{i}+\widehat{\gamma}\left(\int_{\Omega}\left[\sum_{\ell=1}^{n} \tilde{d}_{\ell}\left|u_{\ell}\right|^{\gamma}\right] d x\right) \hat{\rho} \widehat{d}_{i}\left|u_{i}\right|^{\hat{\gamma}-2} u_{i}+\widehat{f}_{i} \quad \text { on } \partial \Omega \text { as } i=1, \ldots, n .
\end{aligned}
$$


Let us introduce the conditions:

$$
\begin{gathered}
(\hat{\rho})^{+} \not \equiv 0 \quad\left(\Longrightarrow V^{+}\left(D_{2}\right) \neq \emptyset(\text { Proposition A.1 and A.2)) }\right. \\
\int_{\partial \Omega} \hat{\rho} d \sigma<0 \quad\left(\Longrightarrow D_{2}(c)<0 \forall c \in R^{n} \backslash\{0\}\right),
\end{gathered}
$$

and let us note that (Proposition 3.3)

$$
(3.104) \Longrightarrow\left(\exists \delta^{*}>0:\left(i_{14}\right) \text { holds if }\left|\lambda_{\ell}\right|,\left|\mu_{\ell}\right| \leq \delta^{*} \text { as } \ell=1, \ldots, n\right) \text {. }
$$

Proposition 3.27. Under assumptions (3.102) and (3.104), if $F \not \equiv 0$ and $\|F\|_{*}$ is sufficiently small, then with $\lambda_{\ell}, \mu_{\ell}$ as in (3.105), system (3.103) has at least one weak solution $\tilde{u}(\tilde{u}=\tilde{\tau} \widetilde{v}, \tilde{\tau}=$ const. $>$ $\left.0, \tilde{v} \in S_{\lambda \mu} \cap V^{+}\left(D_{2}\right)\right)$. When $\gamma<p \leq q_{1}$, it results in

$$
\tilde{u}_{h} \not \equiv 0 \text { even if } F_{h} \equiv 0 \text {. }
$$

Proof. The existence of $\tilde{u}$ is due to ([1], Theorem 3.2). About (3.106), it is sufficiently (Remark 1.1) to verify that

$$
\left(i_{16}^{h}\right) \text { holds as } h=1, \ldots, n \quad \text { with } \mathfrak{F}=S_{\lambda \mu} \cap V^{+}\left(D_{2}\right) .
$$

Let $v=\left(v_{1}, \ldots, v_{n}\right) \in V^{+}\left(D_{2}\right) \cap S_{\lambda \mu}$ with, for example, $v_{1} \equiv 0$. Let $\psi=\sum_{\ell \neq 1} d_{\ell} v_{\ell}$. Let $\mathbb{K} \subseteq \Omega$ be a compact set having positive measure such that

$$
\tilde{d}_{1}>0 \text { in } \mathbb{K} \text { if } \rho d_{1} \psi \equiv 0, \quad \text { either } \rho d_{1} \psi>0 \text { in } \mathbb{K} \text { or } \rho d_{1} \psi<0 \text { in } \mathbb{K} \text { if } \rho d_{1} \psi \not \equiv 0 .
$$

Proposition A.1 lets us choose $\varphi \in C_{0}^{\infty}\left(R^{N}\right)$ satisfying the following conditions:

$$
\begin{gathered}
\delta=p^{-1}\left[\int_{\Omega}|\nabla \varphi|^{p} d x-\lambda_{1} \int_{\Omega} b_{1}|\varphi|^{p} d x\right]>0, \quad \int_{\Omega} \tilde{d}_{1} \varphi^{\gamma} d x>0 \text { if } \rho d_{1} \psi \equiv 0, \\
\int_{\Omega} \rho d_{1}|\psi|^{q_{1}-2} \psi \varphi d x>0 \text { if } \rho d_{1} \psi \neq \equiv .
\end{gathered}
$$

Then with $v(s)=\left((1-s)^{1 / p} \delta^{-1 / p} \varphi, s^{1 / p} v_{2}, \ldots, s^{1 / p} v_{n}\right)$, we have

$$
\begin{gathered}
H_{\lambda \mu}(v(s))=1 \quad \forall s \in[0,1], \quad D_{2}(v(s))>0 \quad \forall s \in\left[s_{0}, 1\right]\left(0 \leq s_{0}<1\right), \\
\lim _{s \rightarrow 1^{-}} \frac{d}{d s} D_{1}(v(s)) \in R, \quad \lim _{s \rightarrow 1^{-}} \frac{d}{d s} D_{2}(v(s))=-\infty \quad \text { if } \rho d_{1} \psi \equiv 0, \\
\lim _{s \rightarrow 1^{-}} \frac{d}{d s} D_{1}(v(s))=-\infty, \quad \lim _{s \rightarrow 1^{-}} \frac{d}{d s} D_{2}(v(s)) \in R \quad \text { if } \rho d_{1} \psi \neq 0 .
\end{gathered}
$$

Now we replace conditions (3.104) with the following:

$$
\hat{\rho} \geq 0, \quad b_{\ell} \geq 0, \quad \widehat{b}_{\ell} \geq 0 \quad \text { as } \ell=1, \ldots, n .
$$


Proposition 3.28. Under assumptions (3.102) and (3.111), if $F \not \equiv 0$ and $\|F\|_{*}$ is sufficiently small, then with $\lambda_{\ell}, \mu_{\ell} \leq 0$ and $\lambda_{\ell}+\mu_{\ell}<0$ as $\ell=1, \ldots, n$ system (3.103) has at least two different weak solution $u^{1}$ and $u^{2}\left(u^{i}=\tau^{i} v^{i}, \tau^{i}=\right.$ const. $\left.>0, v^{1} \in S_{\lambda \mu} \cap V^{+}(F), v^{2} \in S_{\lambda \mu} \cap V^{+}\left(D_{2}\right)\right)$. When $r<p \leq q_{1}$, it results in

$$
u_{h}^{2} \not \equiv 0 \text { even if } F_{h} \equiv 0 \text {. }
$$

Proof. The existence of $u^{1}$ and $u^{2}$ is due to ([1], Theorems 3.1, 3.2, and 3.3; Remark 3.1). Relation (3.112) is proved as in Proposition 3.27.

\section{Appendix}

In this appendix, we present some results used previously. The first one is trivial. The second one is easy to prove. It is possible to show the third one and the fourth one with the technique developed by Drabek in ([7, Lemma 3.2]). The symbols $\sigma, \hat{p}$, and $\tilde{p}$ are the same introduced in Section 3.

Proposition A.1. Let $\Omega$ be an open set of $R^{N}$. Let $\mathbb{K} \subseteq \Omega$ be a compact set with $|\mathbb{K}|_{N}>0$. If $\Omega^{\prime}$ is an open set such that $\mathbb{K} \subseteq \Omega^{\prime} \subseteq \Omega$, then there exists a family of functions $\left(\varphi_{\varepsilon}\right)_{0<\varepsilon<\varepsilon_{0}} \subseteq C_{0}^{\infty}(\Omega)$ such that

$$
\begin{gathered}
0 \leq \varphi_{\varepsilon} \leq 1, \operatorname{supp} \varphi_{\varepsilon} \subseteq \Omega^{\prime}, \quad \varphi_{\varepsilon} \longrightarrow x \text { strongly in } L^{s}(\Omega), \\
\int_{\Omega}\left|\nabla \varphi_{\varepsilon}\right|^{s} d x \longrightarrow+\infty \quad \text { as } \varepsilon \longrightarrow 0^{+} \forall s \in[1,+\infty[,
\end{gathered}
$$

where $X$ is the characteristic function of $\mathbb{K}$.

Proposition A.2. Let $\Omega \subseteq R^{N}$ be an open, bounded, connected and $C^{0,1}$ set. Let $U$ be an open neighborhood of $\partial \Omega$. If $\Gamma$ is a subset of $\partial \Omega$ with $\sigma(\Gamma)>0$, then there exist a compact set $\widehat{\Gamma} \subseteq \Gamma$ with $\sigma(\widehat{\Gamma})>0$ and a family of functions $\left(\varphi_{\varepsilon}\right)_{0<\varepsilon<\varepsilon_{0}} \subseteq C_{0}^{\infty}\left(R^{N}\right)$ such that

$$
\begin{gathered}
0 \leq \varphi_{\varepsilon} \leq 1, \quad \operatorname{supp} \varphi_{\varepsilon} \subseteq U, \quad \varphi_{\varepsilon} \longrightarrow \widehat{x} \text { strongly in } L^{s}(\partial \Omega), \\
\int_{R^{N}} \varphi_{\varepsilon}^{s} d x \longrightarrow 0 \quad \text { as } \varepsilon \longrightarrow 0^{+} \forall s \in[1,+\infty[
\end{gathered}
$$

where $\widehat{x}$ is the characteristic function of $\widehat{\Gamma}$.

Let $\Omega \subseteq R^{N}$ be an open, bounded, connected and $C^{0,1}$ set. Let as $i=$ $1, \ldots, n A_{i}\left(x, \xi, \eta^{1}, \ldots, \eta^{n}\right)$ be a Carathèodory function into $R^{N}$ defined for $x \in \Omega$, for $\xi \in R^{n}$ and for $\left(\eta^{1}, \ldots, \eta^{n}\right) \in\left(R^{N}\right)^{n}$ such that

$$
A_{i}\left(x, \xi, \eta^{1}, \ldots, \eta^{n}\right) \cdot \eta^{i} \geq c_{0}\left|\eta^{i}\right|^{p}
$$

where $1<p<+\infty, c_{0}=$ const. $>0$. 
Proposition A.3. Let $\left(u_{1}, \ldots, u_{n}\right) \in\left(W_{0}^{1, p}(\Omega)\right)^{n}$ with $u_{i} \geq 0$. If there exist $\left.r \in\right] p, \tilde{p}[$ and $g \in$ $L^{r /(r-p)}(\Omega)$ with $g \geq 0$ such that

$$
\begin{array}{r}
\sum_{i=1}^{n} \int_{\Omega} A_{i}\left(x, u_{1}, \ldots, u_{n}, \nabla u_{1}, \ldots, \nabla u_{n}\right) \cdot \nabla v_{i} d x \leq \int_{\Omega} g\left(\sum_{i=1}^{n} u_{i}\right)^{p-1}\left(\sum_{i=1}^{n} v_{i}\right) d x \\
\forall\left(v_{1}, \ldots, v_{n}\right) \in\left(W_{0}^{1, p}(\Omega) \cap L^{\infty}(\Omega)\right)^{n} \text { with } v_{i} \geq 0,
\end{array}
$$

then $u_{i} \in L^{\infty}(\Omega)$ as $i=1, \ldots n$.

Proposition A.4. Let $\left(u_{1}, \ldots, u_{n}\right) \in\left(W^{1, p}(\Omega)\right)^{n}$ with $u_{i} \geq 0$. If there exist $\left.r \in\right] p, \hat{p}[, g \in$ $L^{r /(r-p)}(\Omega)$ with $g \geq 0, \hat{g} \in L^{r /(r-p)}(\partial \Omega)$ with $\widehat{g} \geq 0$ such that

$$
\begin{aligned}
& \sum_{i=1}^{n} \int_{\Omega} A_{i}\left(x, u_{1}, \ldots, u_{n}, \nabla u_{1}, \ldots, \nabla u_{n}\right) \cdot \nabla v_{i} d x \\
& \leq \int_{\Omega} g\left(1+\sum_{i=1}^{n} u_{i}\right)^{p-1}\left(\sum_{i=1}^{n} v_{i}\right) d x+\int_{\partial \Omega} \widehat{g}\left(1+\sum_{i=1}^{n} u_{i}\right)^{p-1}\left(\sum_{i=1}^{n} v_{i}\right) d \sigma \\
& \forall\left(v_{1}, \ldots, v_{n}\right) \in\left(W^{1, p}(\Omega) \cap L^{\infty}(\Omega)\right)^{n} \text { with } v_{i} \geq 0
\end{aligned}
$$

then $u_{i} \in L^{\infty}(\Omega)$ as $i=1, \ldots, n$.

Remark A.5. If $\widehat{g} \equiv 0$, we can suppose $r \in] p, \tilde{p}[$.

\section{Acknowledgment}

This paper is supported by the Second University of Naples.

\section{References}

[1] L. Toscano and S. Toscano, "On the solvability of a class of general systems of variational equations with nonmonotone operators," Journal of Interdisciplinary Mathematics, vol. 14, no. 2, pp. 123-147, 2011.

[2] P. Drábek and S. I. Pohozaev, "Positive solutions for the $p$-Laplacian: application of the fibering method," Proceedings of the Royal Society of Edinburgh. Section A. Mathematics, vol. 127, no. 4, pp. 703-726, 1997.

[3] S. I. Pohozaev and L. Véron, "Multiple positive solutions of some quasilinear Neumann problems," Applicable Analysis, vol. 74, no. 3-4, pp. 363-390, 2000.

[4] A. Anane, "Simplicité et isolation de la première valeur propre du $p$-laplacien avec poids," Comptes Rendus des Séances de l'Académie des Sciences. Série I. Mathématique, vol. 305, no. 16, pp. 725-728, 1987.

[5] P. Tolksdorf, "Regularity for a more general class of quasilinear elliptic equations," Journal of Differential Equations, vol. 51, no. 1, pp. 126-150, 1984.

[6] N.S. Trudinger, "On Harnack type inequalities and their application to quasilinear elliptic equations," Communications on Pure and Applied Mathematics, vol. 20, pp. 721-747, 1967.

[7] P. Drabek, Strongly Nonlinear Degenerated and Singular Elliptic Problems, vol. 343 of Pitman Research Notes in Math, Longman, London, UK, 1996. 


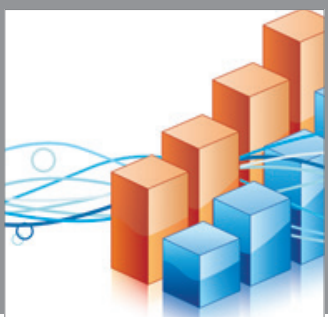

Advances in

Operations Research

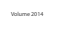

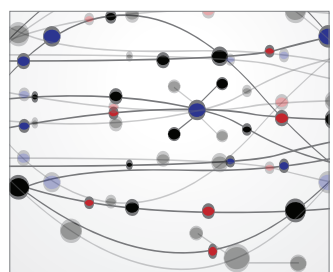

\section{The Scientific} World Journal
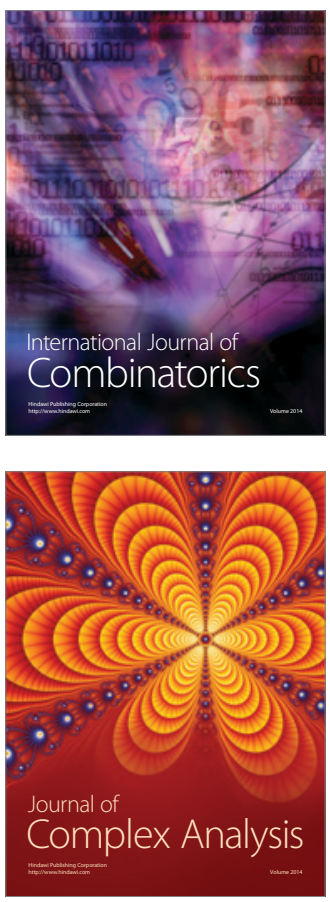

International Journal of

Mathematics and

Mathematical

Sciences
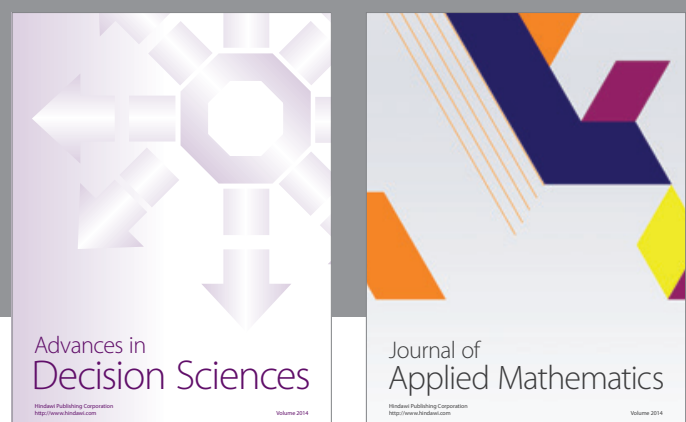

Journal of

Applied Mathematics
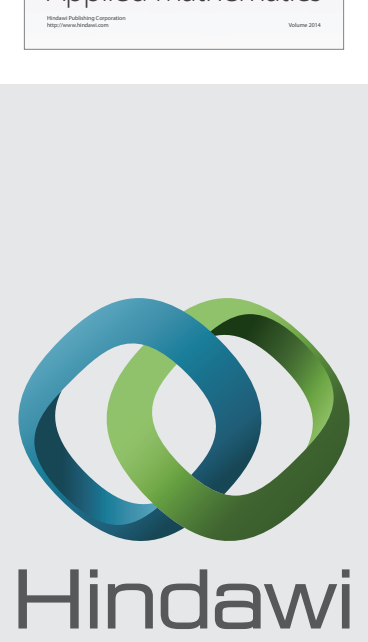

Submit your manuscripts at http://www.hindawi.com
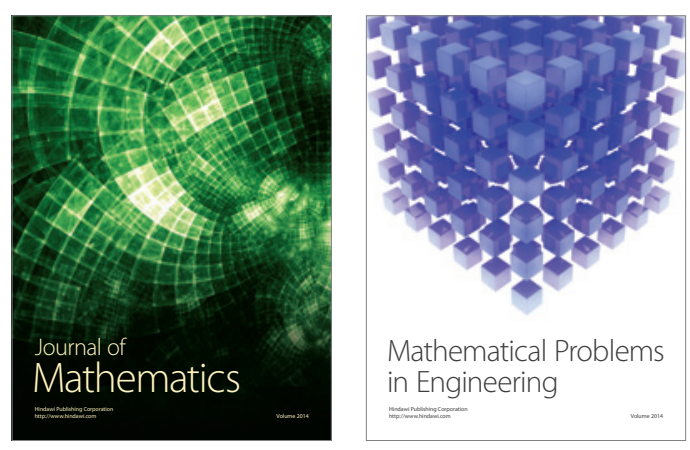

Mathematical Problems in Engineering
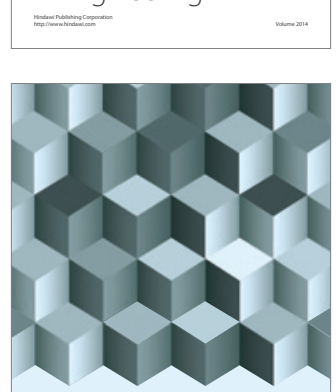

Journal of

Function Spaces
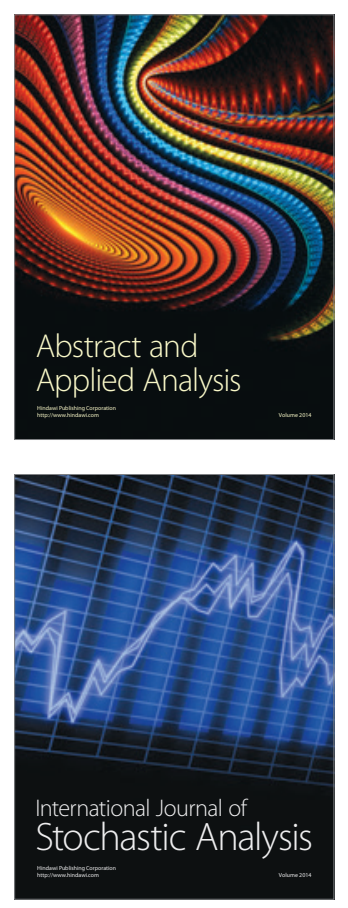

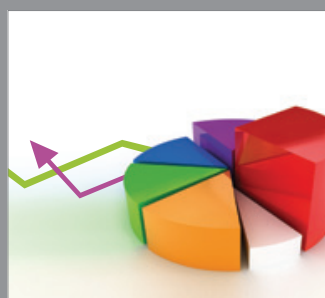

ournal of

Probability and Statistics

Promensencen
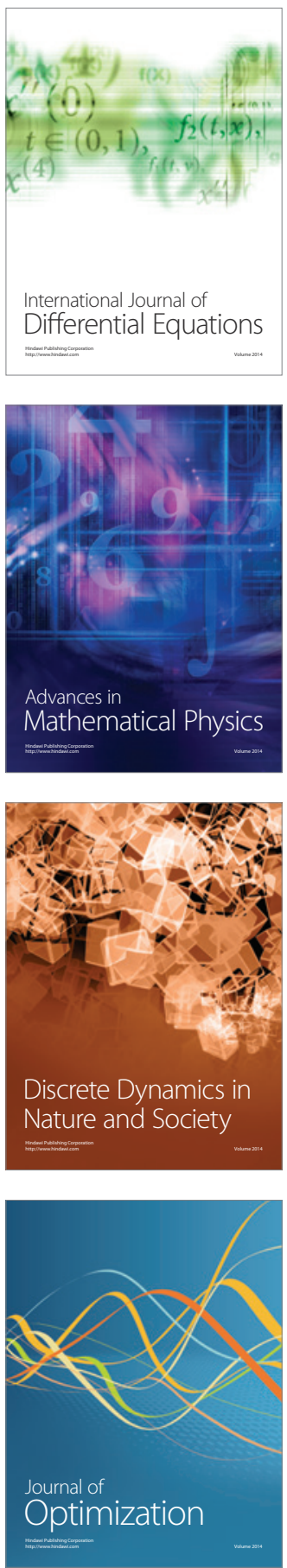